\title{
A Throughput-Centric View of the Performance of Datacenter Topologies
}

\author{
Pooria Namyar \\ University of Southern California
}

\author{
Sucha Supittayapornpong \\ Vidyasirimedhi Institute of Science \\ and Technology
}

\author{
Mingyang Zhang \\ University of Southern California
}

\author{
Minlan Yu \\ Harvard University
}

\begin{abstract}
While prior work has explored many proposed datacenter designs, only two designs, Clos-based and expander-based, are generally considered practical because they can scale using commodity switching chips. Prior work has used two different metrics, bisection bandwidth and throughput, for evaluating these topologies at scale. Little is known, theoretically or practically, how these metrics relate to each other. Exploiting characteristics of these topologies, we prove an upper bound on their throughput, then show that this upper bound better estimates worst-case throughput than all previously proposed throughput estimators and scales better than most of them. Using this upper bound, we show that for expander-based topologies, unlike Clos, beyond a certain size of the network, no topology can have full throughput, even if it has full bisection bandwidth; in fact, even relatively small expander-based topologies fail to achieve full throughput. We conclude by showing that using throughput to evaluate datacenter performance instead of bisection bandwidth can alter conclusions in prior work about datacenter cost, manageability, and reliability.
\end{abstract}

\section{CCS CONCEPTS}

- Networks $\rightarrow$ Data center networks; Network performance modeling; Network manageability; Topology analysis and generation; $\bullet$ General and reference $\rightarrow$ Metrics;

\section{KEYWORDS}

Data centers, Throughput, Clos topologies, Network management

\section{ACM Reference Format:}

Pooria Namyar, Sucha Supittayapornpong, Mingyang Zhang, Minlan Yu, and Ramesh Govindan. 2021. A Throughput-Centric View of the Performance of Datacenter Topologies. In ACM SIGCOMM 2021 Conference (SIGCOMM '21), August 23-28, 2021, Virtual Event, USA. ACM, New York, NY, USA, 21 pages. https://doi.org/10.1145/3452296.3472913

Permission to make digital or hard copies of all or part of this work for personal or classroom use is granted without fee provided that copies are not made or distributed for profit or commercial advantage and that copies bear this notice and the full citation on the first page. Copyrights for components of this work owned by others than ACM must be honored. Abstracting with credit is permitted. To copy otherwise, or republish, to post on servers or to redistribute to lists, requires prior specific permission and/or a fee. Request permissions from permissions@acm.org.

SIGCOMM '21, August 23-28, 2021, Virtual Event, USA

(C) 2021 Association for Computing Machinery.

ACM ISBN 978-1-4503-8383-7/21/08.

https://doi.org/10.1145/3452296.3472913

\author{
Ramesh Govindan \\ University of Southern California
}

\section{INTRODUCTION}

A primary contributor to the success of cloud computing is the datacenter, a warehouse-style agglomeration of compute and storage on commodity servers. The performance of distributed applications running inside a datacenter, like search, reliable storage, and social networks, is strongly determined by the design of the datacenter network. This network consists of a topology in which switches interconnect servers. Today, datacenters routinely have tens of thousands of switches connecting hundreds of thousands of servers. Our focus, in this paper, is on the design and evaluation of topologies for such large-scale datacenters.

Datacenter topology designs. Two distinct classes of topology designs have emerged in recent years. Clos [8] based designs include Fat-tree [1], VL2 [15], Jupiter [42] and Facebook Fabric [3], and failure-resilient variants, such as F10 [36]. These hierarchical designs are bi-regular, in which a switch either connects to $H$ servers, or none at all (Figure 1). More recent alternative designs target lower installation costs and/or incur lower management costs than Clos-based topologies. These designs employ an expander-graph to interconnect switches, and include Jellyfish [44], Xpander [47], and FatClique [52]. These topologies are uni-regular: every switch connects to $H$ servers (Figure 1). In both classes, each server connects to exactly one switch. ${ }^{1}$

Measures of topology capacity. The capacity of the data center network limits the performance of applications it hosts. Intuitively, a topology with enough capacity to permit every server to send traffic at full line rate simplifies cloud application design: operators can place application instances anywhere in the network without impacting performance, and this placement flexibility enables applications to be more cost efficient and more robust to correlated failures (e.g., of an entire rack or power domain) [15, 21, 35, 42].

Most prior work [1, 3, 15, 42, 52] has used the network's bisection bandwidth, the smallest aggregate capacity of the links crossing the worst-case cut among all the cuts that divide the topology graph into two halves, as a measure of its capacity. A topology has full bisection bandwidth if its bisection bandwidth is at least equal to half of the total servers; for Clos-based designs, such a topology permits arbitrary application instance placement.

Other work [24, 26, 27, 50,51] has explored an alternative measure of network capacity, throughput, defined as follows. The throughput under traffic matrix $T$ is the highest scaling factor $\theta(T)$ such that the topology can support the traffic matrix, $T \cdot \theta(T)$,

\footnotetext{
${ }^{1}$ Other topology designs, such as DragonFly [30], and SlimFly [6], do not scale to the sizes of modern data centers, so we do not consider them in this paper; see $§ 7$.
} 

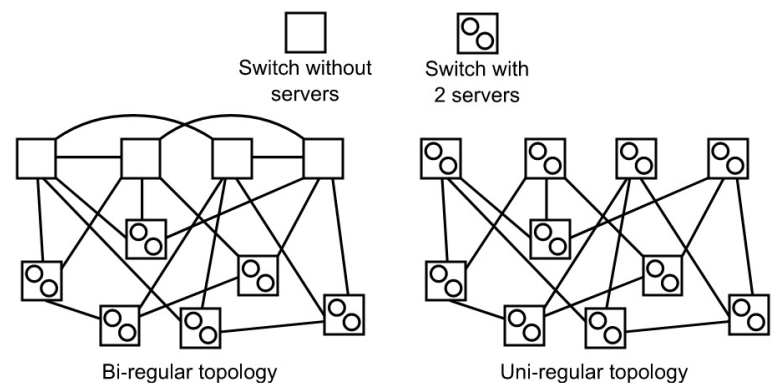

FIgURE 1: Uni-regular and bi-regular topologies.

without violating any link's capacity constraint. The throughput of a topology denoted by $\theta^{*}$ is the worst-case throughput among all traffic matrices. A topology can support any traffic demand if and only if $\theta^{*}$ is at least 1 (in this case, we say the topology has full throughput). Because it can support any traffic demand, a full throughput topology also permits arbitrary application instance placement by definition. ${ }^{2}$

How prior work uses these metrics. These metrics can help evaluate topology design, perform cost comparisons, or assess the complexity of network expansion. As Table 1 shows $\boldsymbol{a}$ substantial body of work has used bisection bandwidth to perform these assessments on large-scale uni-regular and bi-regular topologies. (Some prior work [27, 43, 44, 47] has used throughput to perform some of these assessments, but for much smaller-scale topologies with only a few thousand servers.)

\begin{tabular}{|l|l|l|l|}
\hline Objective & Metric & Topology Class & Prior work \\
\hline \multirow{2}{*}{ Evaluate Design } & \multirow{2}{*}{ BBW } & bi-regular & {$[1,15,42]$} \\
\cline { 3 - 4 } & & uni-regular & {$[44,47,52]$} \\
\hline \multirow{2}{*}{ Assess Cost } & \multirow{2}{*}{ BBW } & bi-regular & {$[15,42,44,52]$} \\
\cline { 3 - 4 } & & uni-regular & {$[44,52]$} \\
\hline \multirow{2}{*}{$\begin{array}{l}\text { Estimate Expansion } \\
\text { complexity }\end{array}$} & \multirow{2}{*}{ BBW } & bi-regular & {$[10,42,52,53]$} \\
\cline { 3 - 4 } & & uni-regular & {$[44,52]$} \\
\hline
\end{tabular}

TABLE 1: Prior work has used bisection bandwidth for large-scale evaluations.

Given this discussion, it is natural to ask: What is the difference between these metrics for uni-regular and bi-regular topologies? Should the papers listed in Table 1 have used throughput instead? How would these assessments change if they did?

To our knowledge, the literature has not explored the precise difference between these two metrics, but has explored related, but slightly different questions. Bisection bandwidth is a graph-cut based metric, and [27] has studied the relation between cut based metrics and throughput at a scale much smaller than those we consider in this paper. As well, [34] shows that the sparsest cut of any topology for a given traffic matrix is within $O(\log N)$ of its throughput for that traffic matrix. Finally, Yuan et al. [50] show that bisection bandwidth cannot predict average throughput of a topology.

\footnotetext{
${ }^{2}$ To actually achieve arbitrary instance placement, one also might need a scalable, practical routing scheme that can exploit the topology's available capacity. For Closbased networks, ECMP-based routing can do so. For large-scale uni-regular topologies, we believe this question is open. We don't address this in this paper since we focus on topology properties.
}

In this paper, we take a first step in understanding the relationship between these metrics by making the following contributions.

Contribution: The Difference Between Full Throughput and Full Bisection Bandwidth for Uni-regular Topologies. We prove (\$4) that for any uni-regular topology, there exists a size (in terms of the number of servers) beyond which the topology cannot have full throughput even if it has full bisection bandwidth. This is true even of small instances of uni-regular topologies with as few as $10-15 \mathrm{~K}$ servers (\$4.2). By contrast, bi-regular Clos topologies are not subject to this limit, and a full bisection bandwidth topology always has full throughput (Figure 2). This means that a topology designer cannot ensure application placement independence (more precisely, the ability to support any arbitrary traffic demand) using a full bisection bandwidth uni-regular topology (Table 1). Put differently, for uni-regular topologies, full bisection bandwidth is necessary but not sufficient to support arbitrary traffic demand; by definition, full throughput is both necessary and sufficient.

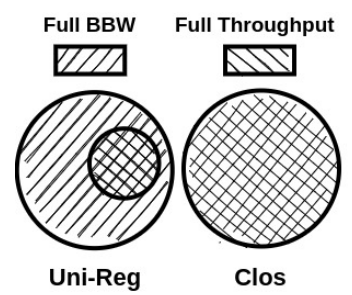

FigURE 2: Full throughput vs. Full bisection bandwidth.

Contribution: A Throughput-Centric View. Table 1 shows that prior work has used bisection bandwidth to evaluate uni-regular and bi-regular topologies; we show that using throughput can lead to different conclusions, impacting cost and management complexity (§5.1). It is also the more appropriate metric: as the previous contribution demonstrates, throughput better captures the capacity of both uni-regular and bi-regular topologies, while bisection bandwidth does not.

- Prior work has argued that a full bisection bandwidth Jellyfish, Xpander or FatClique uses 50\% fewer switches than a full bisection bandwidth Clos [8]. We show that a full throughput Jellyfish [44], Xpander [47] or FatClique [52] uses only $25 \%$ fewer switches than a full throughput Clos. This finding is important, because the smaller cost differential may make uni-regular topologies less attractive relative to Clos (whose packaging and routing simplicity may outweigh its higher cost).

- Prior work has argued that a Jellyfish or FatClique can be expanded: (a) with minor bandwidth loss while keeping the number of servers per switch constant; (b) using a random rewiring strategy [52] simpler than that for Clos [53]. This assumes that bandwidth loss is estimated using bisection bandwidth. We show that, expanding a full throughput Jellyfish or FatClique by even a small amount, while keeping fixed the number of servers per switch, can result in a topology without full throughput. Thus, a designer wishing to maintain full throughput for uni-regular topologies after expansion may need to re-wire servers, requiring a much more complex expansion strategy than Clos. 
- Datacenter designers have traded off topology capacity for lower cost by designing over-subscribed topologies. The FatTree [1] paper defines the over-subscription ratio of a topology as the ratio of the worst-case achievable throughput between end-hosts to the aggregate bisection bandwidth. Our results suggest that, for uniregular topologies, a more direct definition of over-subscription ratio is the throughput itself (a throughput less than 1 indicates an over-subscribed topology). We find that, for these topologies, the bisection-bandwidth based over-subscription ratio overestimates the throughput by up to $50 \%$. Thus, a designer using that definition would build a network whose actual capacity is lower than the targeted capacity.

Contribution: An Efficiently-Computable, Tight, Throughput Upper Bound. The previous contributions require a way to compute the throughput of large uni-regular and bi-regular topologies. To this end, we make the following contributions.

- We prove an upper bound on the throughput of uni-regular and bi-regular topologies $(\S 2)$.

- We empirically show (§3) that this upper bound is tighter and scales better than existing approaches of estimating network capacity or throughput: the throughput bound in [43], heuristics for estimating throughput in $[23,24,51]$, bisection bandwidth, and sparsest cut [27].

- This scalable throughput upper bound can be used to better assess properties of datacenter topologies at larger scales than previously possible, giving a designer greater confidence in a particular topology (§5.2). A concrete example is resilience. Prior work showed that Jellyfish [44] and Xpander [47] degrade gracefully with random link failure for up to $1 \mathrm{~K}$ servers; we show that, for a $131 \mathrm{~K}$ sized Jellyfish or Xpander, degradation is less than graceful (the throughput after failure can be up to $20 \%$ lower than what one might expect with graceful degradation) under random failure.

Ethics. This work does not raise any ethical issues.

\section{AN UPPER BOUND ON THROUGHPUT}

In this section, we prove an upper bound on throughput that applies to uni-regular and bi-regular topologies.

\subsection{Complexity of Computing Throughput Bounds}

A permutation matrix is one in which each row and each column has exactly one non-zero entry. A permutation matrix can indicate traffic either at the server-level (where each entry denotes traffic between two servers), or switch-level. In server-level permutation matrices, all non-zero entries are normalized to 1 while for switchlevel matrices, they are the number of servers connected to the switch $(H)$. In this section, we show that this set of switch-level permutation traffic matrices, denoted by $\hat{\mathcal{T}}$, is sufficient to characterize the throughput of uni-regular and bi-regular topologies.

Notation. Entry $t_{u v}$ of the switch-level traffic matrix $T$ describes the traffic demand from switch $u$ to switch $v$. Let $\mathcal{K}$ be the set of all switches with servers, and $H$ be the number of servers connected to each switch in $\mathcal{K}$. To determine the throughput of the topology, we

\begin{tabular}{|c|l|}
\hline Notation & Description \\
\hline \hline$N$ & Total number of servers \\
$E$ & Total number of switch-to-switch links \\
$R$ & Switch radix \\
$H$ & Number of servers per switch \\
$\mathcal{S}$ & Set of switches with and without servers \\
$\mathcal{K}$ & Set of switches with $H$ servers $(\mathcal{K} \subseteq \mathcal{S})$ \\
$t_{u v}$ & Traffic demand from $u$ to $v$ where $u, v \in \mathcal{K}$ \\
$T=\left[t_{u v}\right]$ & $|\mathcal{K}| \times|\mathcal{K}|$ traffic matrix with demands $t_{u v}$ 's \\
$\mathcal{T}$ & Saturated hose-model set \\
$\hat{\mathcal{T}}$ & Permutation traffic set \\
$\theta(T)$ & Throughput under traffic matrix $T$ \\
$\theta^{*}$ & Topology throughput $\left(\theta^{*}=\min _{T \in \mathcal{T}} \theta(T)\right)$ \\
$L_{u v}$ & Shortest path length from switch $u$ to $v$ \\
\hline
\end{tabular}

TABLE 2: Notation

use the hose model $[11]^{3}$, where every switch sends and receives traffic at no more than its maximum rate $H$ (for simplicity, each link has unit capacity). The hose-model traffic set is the set of traffic matrices that conform to the hose model:

$$
\left\{T \in \mathbb{R}_{+}^{|\mathcal{K}| \times|\mathcal{K}|:}: \begin{array}{ll}
\sum_{u \in \mathcal{K}} t_{u v} \leq H & \forall v \in \mathcal{K} \\
\sum_{v \in \mathcal{K}} t_{u v} \leq H & \forall u \in \mathcal{K}
\end{array}\right\}
$$

where $\mathbb{R}_{+}$is the set of non-negative reals. This traffic set includes the commonly-used traffic matrices such as all-to-all and random permutations, and it applies not just to uni-regular topologies, but to bi-regular topologies as well. A bi-regular topology contains two types of switches: one without attached servers, and one in which each switch has $H$ servers. Switches without servers can not source or sink any traffic, and as a result, it suffices to describe the traffic matrix only by switches with attached servers $(\mathcal{K})$.

Our hose model definition is consistent with [27], which bases its definition on server-level traffic matrices. Our definition uses switch-level traffic matrices, leveraging the fact that uni-regular and bi-regular topologies have $H$ servers per switch and each server connects to exactly one switch.

On computing the throughput of a topology. Since the hosemodel traffic set contains an infinite number of traffic matrices, computing the throughput of the topology (the minimum throughput across all traffic matrices) is intractable.

To improve the tractability, consider the following set of traffic matrices that we call the saturated hose model set, $\mathcal{T}$, where each switch sends and receives traffic at exactly its maximum rate $H$ :

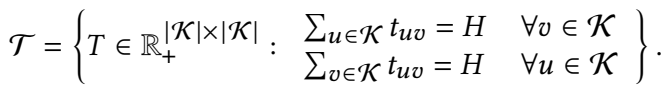

This set dominates the hose-model traffic set, since we can always augment any hose-model traffic matrix with a non-negative value to produce a saturated hose-model traffic matrix. So, the minimum throughput across all traffic matrices in the hose model set cannot be smaller than the minimum throughput across all traffic matrices in $\mathcal{T}$. However, there are still infinitely many elements in $\mathcal{T}$. The following theorem shows that for uni-regular and bi-regular

\footnotetext{
${ }^{3}$ In the hose model, the end-host traffic rate is bounded by the port speed, which means the model only permits admissible traffic patterns for the topology. Our use of the hose model is consistent with prior work [11,27].
} 
topologies, it suffices to consider an even smaller traffic set in order to compute throughput.

THEOREM 2.1. The throughput of a uni-regular or a bi-regular topology is the minimum throughput across all traffic matrices in the permutation traffic set $\hat{\mathcal{T}}$.

Proof Sketch. §A contains the detailed proof, which proceeds in two steps. First, it shows that $\hat{\mathcal{T}}$ represents the extrema of the convex polytope formed by the traffic matrices in $\mathcal{T}$. Second, relying on the convexity of the set $\mathcal{T}$, it shows that the minimum throughput across all traffic matrices must correspond to a permutation traffic.

Prior work [45] has used a similar convexity argument in a slightly different context, and [46] proves a similar theorem in a more limited context (for oblivious routing). Other prior work ([29], Conjecture 2.4) has stated Theorem 2.1 as a conjecture.

The size of $\hat{\mathcal{T}}$, while finite, grows combinatorially with the matrix dimension, so it is still infeasible to iterate over all its elements in order to compute throughput. However, in any traffic matrix in $\hat{\mathcal{T}}$, each switch $u$ sends traffic at full rate to exactly one other switch $v$. We exploit this, together with the structure of uni-regular and biregular topologies to derive an efficiently computable upper bound on the throughput of these topologies (§2.2).

\subsection{Throughput Upper Bound}

We now use Theorem 2.1 to derive a closed-form expression for the upper bound on the throughput of a uni-regular or a bi-regular topology. Throughput is both a function of the topology and the routing algorithm used to route traffic demands; the derived upper bound is independent of the routing algorithm.

Upper bound for uni-regular topology throughput. The following theorem establishes a tractable closed-form expression for the throughput of a uni-regular topology. It assumes, without loss of generality, a uni-regular topology with $H$ servers per switch, and unit link capacity.

THeorem 2.2. The maximum achievable throughput for a uniregular topology, under any routing, is bounded by:

$$
\theta^{*} \leq \min _{T \in \hat{\mathcal{T}}} \frac{2 E}{H \sum_{(u, v) \in \mathcal{K}^{2}} L_{u v} \mathbb{I}\left[t_{u v}>0\right]}
$$

where $E$ is the number of switch-to-switch links in the topology, $L_{u v}$ is the shortest path length from switch $u$ to switch $v$ and $\mathbb{I}[\cdot]$ is an indicator function.

Proof SкETCh. §B contains the detailed proof, which relies on the optimal solution of the path-based multi-commodity flow problem $(\S \mathrm{H}$, commonly used in wide-area network traffic engineering [33]). For a given traffic matrix $T$, path-based multi-commodity flow maximizes throughput $\theta(T)$. Now, consider an arbitrary switch $u$. Its total ingress traffic consists of two components: the traffic destined to its servers, which depends on $\theta(T)$, and its transit traffic We upper-bound the ingress traffic by the aggregate link capacity at the switch, and lower-bound it by the total transit traffic derived from the path lengths and the flow split ratios. Solving these inequalities, and applying Theorem 2.1 gives Equation 1.
Efficiently computing the throughput bound. The RHS of Equation 1 chooses a permutation traffic matrix that maximizes total path length. Finding this matrix is equivalent to finding nearworst-case traffic matrix in [27]. In that work, the authors present an intuitive form of the throughput upper bound and suggest an intuitive heuristic for constructing a "difficult" server-level traffic matrix (near-worst-case). In this paper, we formally prove the throughput upper bound and use a slightly different approach (discussed below) that constructs a switch-level traffic matrix to achieve the minimum of the RHS of Equation 1.

To find the minimum throughput, we construct a complete bipartite graph $B$ (consisting of two disjoint set of nodes $U$ and $V$ ) from the given topology G. $U$ and $V$ represent all the possible source and destination switches with directly connected servers in $G$ respectively. The weight of the edge $(u, v)$ where $u \in U$ and $v \in V$ is the shortest path length from switch $U$ to switch $V$. The permutation traffic matrix that determines the throughput bound in Equation 1 corresponds to the weighted maximum matching in $B$. We call this the maximal permutation matrix.

Extension to bi-regular topologies. Theorem 2.2 applies to biregular topologies as well. Intuitively, additional switches with no servers increase capacity for transit traffic which is reflected in the numerator of Equation 1. We prove this formally in $\S \mathrm{C}$. The theorem also applies to uni-regular and bi-regular topologies in which each switch $u$ has a different radix $R_{u}$; we have omitted the description of this extension for brevity.

Theorem 2.2 implies that throughput of a topology is proportional to total link capacity and inversely proportional to maximal total path length of the maximal permutation matrix. Prior work [43] has computed an upper-bound on the average throughput of uni-regular topologies across all uniform traffic matrices (the all-to-all and permutation matrices). In contrast, we bound the worst-case throughput, and our bound is significantly closer (§3.2) to the worst-case behavior of uni-regular topologies at all scales than the bound of [43]. Our bound is also more general: it applies to bi-regular topologies as well, and across all traffic matrices (as a consequence of Theorem 2.1).

On server-level vs. switch-level traffic matrices. We exploit the regularity in uni-regular and bi-regular topologies and reason about switch-level permutation traffic matrices, rather than server-level ones. This helps us efficiently compute the upper-bound even for large topologies (§3). This efficiency does not impact the throughput estimate, relative to using a server-level permutation matrix, as we now show.

If we had used the server-level TMs, the throughput upper-bound would have been the same. A switch-level maximal permutation matrix $\hat{T}$, when converted to server-level $\hat{T}_{n}$, is a solution to the corresponding server-level weighted maximum matching problem. We can prove this by contradiction. Let, for any server $u, s(u)$ be the switch connected to $u$ and assume that $\hat{T}_{n}$ can be improved by (the total path length of the permutation matrix can be increased by, see denominator of Equation 1$)$ a set of actions on $(u, v)(e . g$., insertion or deletion of a flow). We can show that $\hat{T}$ can be also improved by the same amount by a similar set of actions on $(s(u), s(v))$. This is because the link from the server to its directly connected 


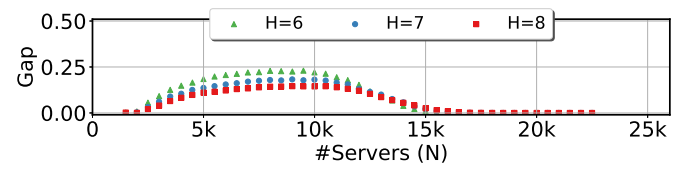

(a) Jellyfish $(\mathrm{K}=100)$

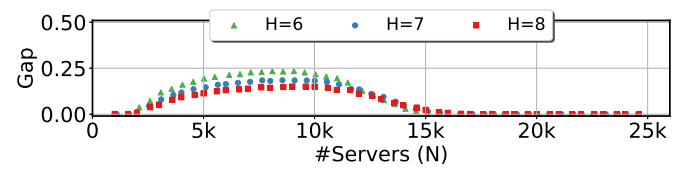

(b) Xpander $(\mathrm{K}=100)$

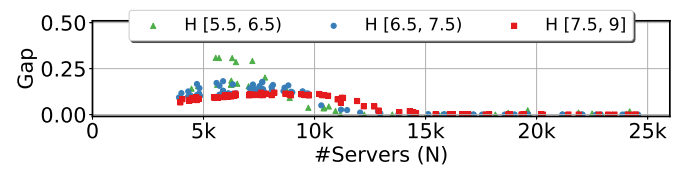

(c) FatClique $(\mathrm{K}=200)$

FigURE 3: Throughput bound vs K-shortest paths Multi-commodity flow. Gap approaches zero as the number of servers $(N)$ increases for all choices of uni-regular topologies and servers per switch $(H)$.

switch does not constrain throughput, so all $L_{u v}$ S do not include it. Thus, adding/removing $(s(u), s(v))$ increases/decreases the total path length by the same amount as adding/removing $(u, v)$ does. This is a contradiction since we assumed $\hat{T}$ is the maximal permutation matrix.

However, the actual throughput of the topology under switchlevel maximal permutation matrix is always less than or equal to the server-level one. If the server-level maximal permutation matrix, when converted to switch-level, is not a permutation matrix, a similar line of proof as Theorem 2.1 can show that the corresponding switch-level traffic matrix is a convex combination of some switch-level permutation traffic matrices. So, at least one of the switch-level permutation matrices has lower throughput than this TM. Hence, considering switch-level matrices not only improves the scalability of our throughput bound but also better captures the minimum throughput of the topology.

\section{EVALUATING THE THROUGHPUT UPPER BOUND}

In this section, we show that throughput upper bound (abbreviated TUB) (a) accurately estimates the worst case throughput and (b) all previously proposed throughput estimators [23, 24, 43, 51] produce worse estimates for uni-regular topologies and most scale poorly. ${ }^{4}$

\subsection{Throughput Gap}

In this section, we compute the throughput gap between the throughput upper bound (abbreviated TUB) and the throughput

\footnotetext{
${ }^{4}$ Our code is available at https://github.com/USC-NSL/TUB
}

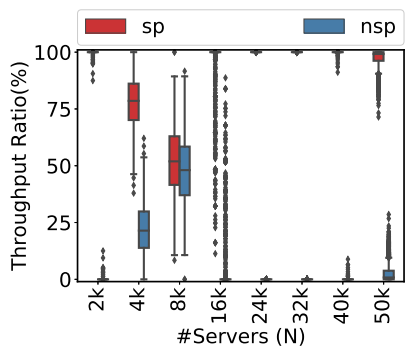

(a) Throughput distribution

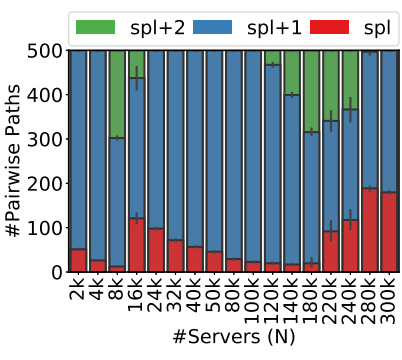

(b) Path distribution
Figure 4: Jellyfish $(\mathrm{H}=8)$. (a) Throughput gap appears at topology sizes that shortest paths does not provide enough diversity. (b) The number of pairwise shortest paths in the maximal permutation matrix periodically increases and decreases. $(\mathrm{sp}=$ shortest path, $\mathrm{nsp}=$ non-shortest path, $\mathrm{spl}=$ shortest path length)

achieved by routing a "worst-case" traffic matrix, and show that this gap is small.

Methodology. Prior work [27] has shown that maximal permutation matrix can achieve worst-case throughput. We have independently verified this. For small topologies, we exhaustively compared the throughput of every TM under KSP-MCF, and the maximal permutation matrix achieves the lowest throughput. For large topologies, we compared the throughput of the maximal permutation matrix with 20 random permutations, and observed that the throughput of maximal permutation matrix is constantly lower, and the gap between these two increases with scale.

To demonstrate that the throughput gap is small, we need to select a routing scheme. We have found that it suffices to solve a path-based multi-commodity flow [33] over K-shortest paths (KSP$\mathrm{MCF}$, see $\S \mathrm{H})$. To compute the throughput gap, we sweep values of $K$ until increasing $K$ does not increase throughput ${ }^{5}$; in most cases, $K=100$ suffices to match тUв. As an aside, we do not mean to suggest that KSP-MCF is practical for large networks; especially for uni-regular topologies, finding a scalable routing scheme that can achieve high throughput is an open question left to future work.

Other details. For all results in the paper, we use METIS [28] to (over) estimate bisection bandwidth, Gurobi [18] to solve linear programs for MCF, the networkx [19] implementations of $K$-shortest paths [49] and the igraph [9] implementation of maximum bipartite matching [32, 40]. FatClique deviates slightly from our definition of uni-regular topologies: in a FatClique topology, $H$ can differ by 1 across switches. We have adapted тUB and the maximal permutation algorithm to deal with this deviation (§I).

For Uni-regular Topologies. Figure 3 shows the throughput gap for тUB for the three uni-regular topologies, for different $H$.

Jellyfish. Figure 3(a) shows the throughput gap for $K=100$ for Jellyfish with $H=8$ (other values of $H$ are qualitatively similar). The gap is non-zero at small scales between $3 \mathrm{~K}-15 \mathrm{~K}$. However, for larger instances, the gap is close to zero.

TUB is loose in the range $3 \mathrm{~K}-15 \mathrm{~K}$ because (a) the proof of Theorem 2.2 uses the observation that throughput is highest when

${ }^{5} \S \mathrm{J}$ shows the results for different values of $K$ 
all paths between each source-destination pair are shortest paths and (b) topologies in this size range have fewer shortest paths, so KSP-MCF routes traffic over non-shortest paths. (Figure 4(a) plots the distribution of the fraction of flows over shortest and non-shortest paths for different topology sizes).

Interestingly, topologies with $100 \mathrm{~K}-180 \mathrm{~K}$ servers have a smaller fraction of shortest paths (Figure 4(b)), so we expect TUB to be loose in that range (we cannot confirm this because KSP-MCF does not scale to those sizes), but expect the throughput gap to be small beyond that range because the fraction of shortest paths increases. However, in $\S \mathrm{E}$, we show that the maximum possible throughput gap approaches zero asymptotically. Future work can explore better throughput bounds that exploit diversity in non-shortest paths.

Xpander and FatClique. Figure 3 shows the throughput gap for Xpander and FatClique, for different values of $H$. Like Jellyfish at $H=8$, the gap is significant at small scales between $5 \mathrm{~K}-15 \mathrm{~K}$ for these topologies and the gap is close to zero for larger instances.

Bi-regular Topologies. For Clos-based bi-regular topologies, ECMP is able to achieve (close to) full throughput (modulo differences in flow sizes [15]). We find that TUB's estimate is also 1 for different Clos topologies, showing that the gap is zero for them as well (Table A.1).

\subsection{Comparison with other throughput metrics}

Prior work has proposed other ways of estimating throughput. For uni-regular topologies, we expect тUB to be (a) faster and (b) more accurate than these other methods, because it leverages properties of uni-regular topologies. In this section, we validate this intuition

Efficiently computing TUB. Before doing so, we briefly discuss some empirical results for the speed of computing тUв. The bottleneck in this computation is the weighted maximum matching in a complete bipartite graph. Several network analysis tools such as networkx [19] and igraph [9], have an efficient implementation of weighted maximum matching. Furthermore, our computation scales well because we abstract the server-level traffic into a switch-level traffic matrix, so that the number of nodes in the constructed bipartite graph reduces significantly. On a machine with 64GB of RAM, we were able to find the throughput upper bound for topologies with up to $180 \mathrm{~K}$ servers with $H=8$ within 20 minutes. For calibration, on the same platform, computing the throughput for routing a permutation traffic matrix using KSP-MCF does not scale beyond $50 \mathrm{~K}$ servers, and using full-blown MCF does not scale beyond $8 \mathrm{~K}$ servers.

Comparison alternatives. Prior work [27] has compared throughput (i.e., the solution to MCF) with cut-based metrics, such as sparsest-cut (using an eigenvector based optimization in [26]) and bisection bandwidth, and [43] computes an upper bound on average throughput of uni-regular topologies across uniform traffic matrices. In addition to these, we compare our method to two other throughput estimators developed for general graphs. Hoefler's method [51] divides a flow into sub-flows on each path between source and destination, and splits the capacity of a link equally across all flows traversing it. Jain's method [24] incrementally routes flows on each path; at each step it allocates residual capacity on a link to all new flows added to the link at this step and iterates until no paths remain.

Results. Figure 5 compares TUB against these alternatives, for Jellyfish topologies with 8 servers per switch. Results for other topologies are similar (omitted for brevity).

Small to medium scale. Figure 5(a) shows the throughput gap (determined using the methodology described in §3.1) for topologies with up to $25 \mathrm{~K}$ servers. TUB has the smallest throughput gap across all alternatives. In the range $15 \mathrm{~K}-25 \mathrm{~K}$, TUB's throughput gap is zero, that of others is higher than 0.2 , and sometimes as high as 0.4 . To illustrate why it is important to have a small throughput gap, consider a scenario in which a network operator wishes to design a full throughput topology; if she uses a loose throughput estimator, the resulting topology may not actually have full throughput.

Moreover, TUB is among the most efficient of the alternatives (Figure 5(b)).

It is both more accurate, and faster than Jain's method (JM) and Hoefler's method (HM). These have large throughput gaps at larger topology sizes (Figure 5(a)). JM and HM exploit edges of each available path, but their estimates are loose because they assume all the sub-flows going through each edge get a fair share of the edge's capacity. This assumption may not maximize the throughput of a traffic matrix; to do this, flows that currently have lower throughput should get more share of the available capacity. JM and HM are a few orders of magnitude slower than тUB (Figure 5(b)) because they exploit more of the topological structure.

Bisection bandwidth and [43] scale better than TUB, but their estimates have large error. Bisection bandwidth is a loose cut-based estimate of throughput as shown by [27] at small scales, and proven by us in $§ 4$. Figure 5(a) empirically verifies this at much larger scales than [27]. Computing exact bisection bandwidth for general networks is intractable [4], so we use a fast heuristic [28] that approximates the bisection bandwidth. Furthermore, the bound in [43] relies on average distance among all the pairs of switches, based on the fact that every switch splits its traffic equally and sends to all the other switches in the average case. Our bound, however, considers structural properties (e.g., distance between individual pairs) to maximize the congestion by routing the traffic between pairs with the largest distance. Therefore, the gap for TUB is smaller than that for [43], but TUB is slower since it considers more details about the topology.

Large scale. Figure 5(c) plots the bisection bandwidth, and the throughput estimated by TUB, and by [43], for topologies for up to $300 \mathrm{~K}$ servers. At these scales, we cannot compute KSP-MCF to estimate the throughput, so we depict the absolute throughput values. [43]'s throughput estimate is consistently and considerably higher across the entire range compared to TuB's. The latter's computational complexity is comparable to that of [43], except for the range $200 \mathrm{~K}-280 \mathrm{~K}$ where тUB exhibits a non-monotonic behavior. тUв attempts to choose disjoint pairs of switches with large distances from each other to construct the maximal permutation matrix, but in topologies of this size range, there are fewer of these pairs with longest possible distance (i.e., diameter), so it takes longer for the 


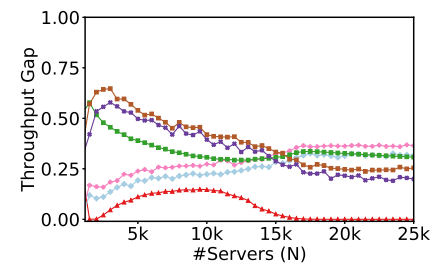

(a) Accuracy

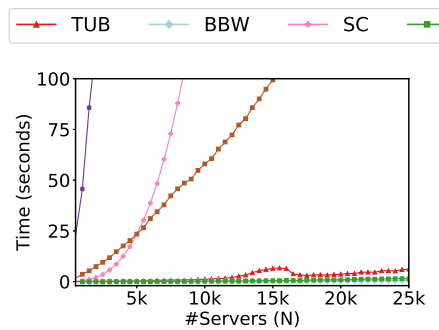

(b) Efficiency

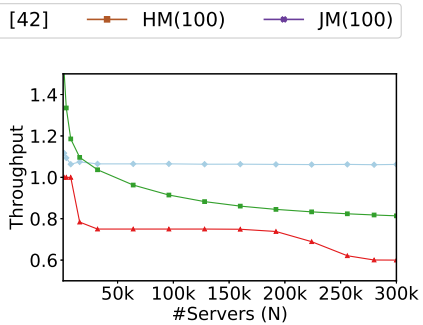

(c) Accuracy, Large Scale

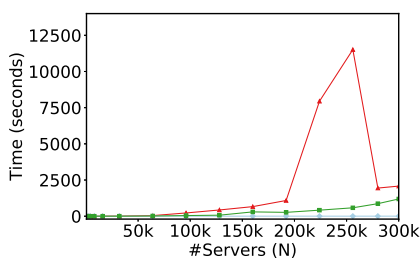

(d) Efficiency, Large Scale

FIGURE 5: TUB is more accurate compared to all the other metrics and almost as fast as bisection bandwidth and throughput bound in [43]. (BBW is bisection bandwidth, SC is sparsest cut, $\mathrm{HM}($.$) is Hoefler's method and JM(.) is Jain's method in which (.) is the number of paths)$

algorithm to search for these disjoint pairs. We expect to significantly reduce the search by parallelizing the weighted maximum matching implementation; we have left this to future work.

Summary. TUB's throughput gap is smaller than those of prior estimators and scales to up to $300 \mathrm{~K}$ servers. This enables us to revisit whether prior evaluations of large-scale topologies using bisection bandwidth would yield different conclusions if throughput were used instead (§5).

\section{LIMITS ON THE THROUGHPUT OF UNI-REGULAR TOPOLOGIES}

In this section, using Theorem 2.2 we establish asymptotic limits on the size of full-throughput uni-regular topologies. Then, exploiting TUB’s scalability and tightness ( 33$)$, we establish practical limits on the size of full-throughput uni-regular topologies for different values of $H$.

\subsection{Asymptotic Limits}

A throughput upper bound for all uni-regular topologies. Theorem 2.2 determines an upper-bound on the throughput for $a$ given uni-regular or bi-regular topology, independent of routing. The following theorem, which applies only to uni-regular topologies, establishes an upper-bound on the throughput across all uniregular topologies, independent of routing.

THEOREM 4.1. The maximum achievable throughput of any uniregular topology with $N$ servers, switch radix $R$ and $H$ servers per switch under any routing is:

$$
\theta^{*} \leq \frac{N(R-H)}{H^{2} D}
$$

where;

$$
D=d\left(\frac{N}{H}-1\right)-\frac{R-H}{R-H-2}\left(\frac{(R-H-1)^{d}-1}{R-H-2}-d\right)
$$

and $d$ is the minimum diameter required to accommodate $\mathrm{N} / \mathrm{H}$ switches computed using Moore bound [39].

Proof Sketch. §D contains the detailed proof. We observe from Equation 1 that throughput is lowest for switch pairs $(u, v)$ for whom the shortest path length $L_{u v}$ is high. Our constructive proof

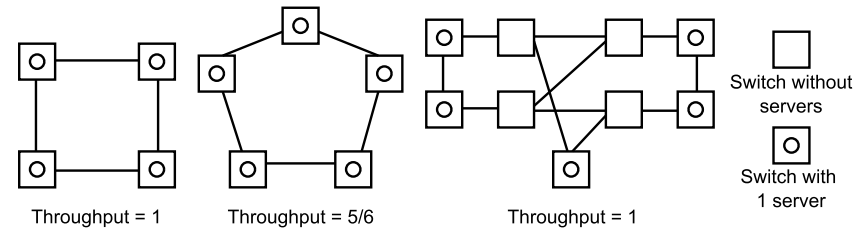

Figure 6: uni-regular topologies can have limited throughput.

first bounds the number of switches whose distance is at least $m$ from a given switch (Lemma 8.1 in $§ \mathrm{D})$. Then, we construct (Algorithm 1 in the Appendix) the maximal permutation traffic matrix in which each switch exchanges traffic with other switches that are furthest from it (Lemma 8.2 in §D). This construction maximizes $L_{u v}$, and from this construction and using Lemma 8.1, we can bound the number of communicating switch pairs whose distances are at least $m$ hops of each other. The bound applies to the denominator of the RHS of Theorem 2.2, resulting in a throughput upper bound independent of the traffic matrix (Lemma 8.3 in $\S \mathrm{D})$.

This theorem formalizes the intuition captured in Figure 6. Fundamentally, a uni-regular topology is constrained by the fact that every switch has to have $H$ servers. The figure shows topologies in which 3-port switches have (at most) $H=1$ servers. The leftmost 4-switch topology has full throughput. However, the addition of a single switch (the middle topology) drops throughput significantly. To recover full throughput in this setting, we need to add four more switches with no servers; these provide additional transit capacity. Figure 7 shows the worst-case TM for the middle topology along with the optimal routing of the TM. It also presents the throughput of the same TM on the bi-regular topology with 4 additional switches.

Relationship between bisection bandwidth and throughput. Using Theorem 4.1, we can derive a necessary condition for any full throughput uni-regular topology:

$$
D \leq \frac{N(R-H)}{H^{2}}
$$

Unlike bi-regular topologies where Clos topologies have full bisection bandwidth and full throughput (see below), uni-regular 


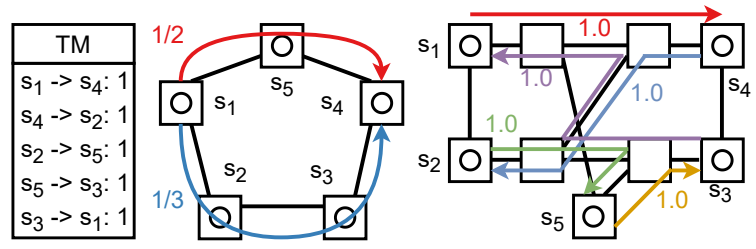

FIGURE 7: The uni-regular topology can support the given worst-case permutation traffic matrix with throughput $=\frac{5}{6}$ while the bi-regular topology with 4 additional switches can support the TM at full throughput. In the uni-regular topology setup, the optimal routing is the following: $\frac{1}{2}$ of each flow is routed through the shortest path while $\frac{1}{3}$ of each flow is routed through the non-shortest path.

\begin{tabular}{|c|c|c|c|c|}
\hline Topology & Condition & $H=8$ & $H=7$ & $H=6$ \\
\hline Uni-regular & Equation 3 & $111 \mathrm{~K}$ & $256 \mathrm{~K}$ & $3.97 \mathrm{M}$ \\
Jellyfish & Full-BBW & $>20 \mathrm{M}$ & $>20 \mathrm{M}$ & $>20 \mathrm{M}$ \\
Xpander & Full-BBW & $>20 \mathrm{M}$ & $>20 \mathrm{M}$ & $>20 \mathrm{M}$ \\
FatClique & Full-BBW & $>20 \mathrm{M}$ & $>20 \mathrm{M}$ & $>20 \mathrm{M}$ \\
\hline
\end{tabular}

TABLE 3: Maximum number of servers, each topology set up can support without violating the condition.

topologies can have full bisection bandwidth, but not full throughput (as illustrated in Figure 2). Table 3 shows the maximum number of servers each topology family can support without violating Equation 3 (switch radix $R$ is 32). It shows that the largest full throughput uni-regular topology with 8 servers per switch can only support $111 \mathrm{~K}$ servers, while the largest full bisection bandwidth Jellyfish, Xpander, or FatClique topologies can support over 20M servers! (In Table 3, for all uni-regular topologies, we were unable to estimate the bisection bandwidth for topologies larger than $20 \mathrm{M}$ servers because of computational limits.)

Scaling limits on uni-regular topologies. Another way of stating the results in Table 3 is that no uni-regular topology with $H=8$ and more than $111 \mathrm{~K}$ servers can have full throughput. This implies that there is a bound on the number of servers that a full throughput uni-regular topology can have. Corollary 1 formalizes this; we prove it in $\S$.

COROLlary 1. For a given switch radix $R$ and servers per switch $H$, there exists a $N^{*}(R, H)$ such that for $N \geq N^{*}(R, H)$, no full throughput uni-regular topology exists with $N$ servers, switch radix $R$ and $H$ servers per switch.

Every Clos-based topology always has full throughput. In contrast to these scaling limits for uni-regular topologies, a fullydeployed Clos-based topology always has full throughput. In §2.1, we observed that Theorem 2.1 applies to Clos-based topologies. Prior work has shown that a multi-stage Clos can (re-arrangeably) support every permutation traffic matrix $[25,41]$. Since Clos is a bi-regular topology, it must have a throughput of 1 because, by Theorem 2.1 , it suffices to consider only permutation traffic matrices to compute the throughput, and Clos can support all permutation traffic matrices (i.e., for each matrix in $\hat{\mathcal{T}}$, Clos has a throughput of
1). Thus, bi-regular topologies like VL2 [15] and FatTree [1], being Clos topologies, have full throughput. We conjecture that F10 [36] also has full throughput (F10 uses a different striping than Clos), but have left it to future work to prove that.

\subsection{The Full-Throughput Frontier}

Table 3 shows the largest possible number of servers any uni-regular topology can support at full throughput. However, this bound is loose in part because it applies generically to all uni-regular topologies. In this section, for each topology family, we characterize, as a function of $H$, the largest size beyond which no topology has fullthroughput ${ }^{6}$ (as estimated by TUB). We call this the full-throughput frontier. For calibration, we also draw the full bisection-bandwidth frontier, defined similarly. This comparison helps us quantitatively understand the Venn diagram of Figure 2.

Methodology. To compute these frontier curves, we generate topologies from each topology family, for different $H$ and $N$. For Jellyfish and Xpander, there is a uniquely defined topology given $H$ and $N$. (In our experiments, we have assumed a fixed switch radix of 32 unless otherwise mentioned.) For each value of $H$, we use binary search on the total number of servers to find the maximum $N$ that provides full bisection bandwidth, or full throughput.

For FatClique, we cannot precisely estimate the full-throughput frontier since its topology instances can be non-monotonic with respect to throughput. Specifically, because of the way it is constructed, for a given $H$, a topology with $N$ servers can have full throughput, but a topology with $N^{\prime}<N$ servers may not. For this reason, for FatClique, we generate a large number of instances for each $H$ and for each, we evaluate whether that instance has both full bisection bandwidth and full throughput, or only full bisection bandwidth.

Results. Figure 8 shows the results of these experiments for Jellyfish, Xpander, and FatClique.

Jellyfish and Xpander. Figure 8(a) shows the full-throughput and full-bisection bandwidth frontier curves for Jellyfish, and Figure 8 (b) for Xpander. For both Jellyfish and Xpander, there is a large gap between these curves; there are many topologies that have full bisection bandwidth, but do not have full throughput. In some configurations (specifically $H$ of 7 and 8), these topologies cannot achieve full throughput even with $10 \mathrm{~K}-15 \mathrm{~K}$ servers. At $H$ of 9 , these topologies can support a few hundred servers with full throughput. For $H$ of 6, Jellyfish and Xpander can support full throughput up to $225 \mathrm{~K}$ servers (off-scale in Figure 8(a), Figure 8(b)).

How does throughput degrade beyond the frontier? At 7 servers per switch, a Jellyfish with $13 \mathrm{~K}$ servers has a TUB of 1 , with $15 \mathrm{~K}$ servers a тUB of 0.94 , and with $17 \mathrm{~K}$ servers a тUв of 0.89 . Similar results hold for Xpander. This appears to suggest that the throughput of these topologies degrade gracefully beyond the frontier, but we have left a more detailed analysis to future work.

FatClique. Because FatClique instances can be non-monotonic with respect to throughput, the full-throughput frontier curve is approximately the boundary separating the blue (Throughput) points from the red (BBW) points in Figure 8(c). Like Jellyfish and

\footnotetext{
${ }^{6}$ Some topologies smaller than this size may also not have full throughput because TUB is an upper bound.
} 


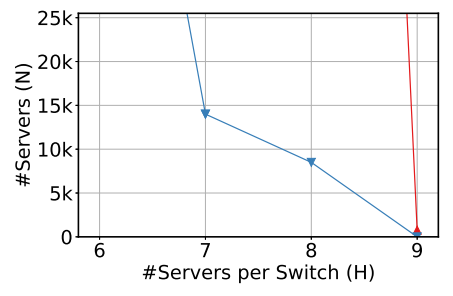

(a) Jellyfish

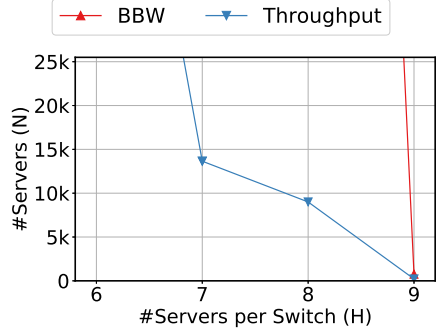

(b) Xpander

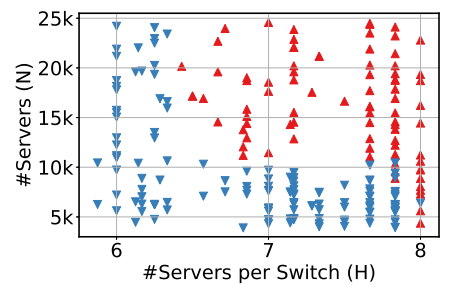

(c) FatClique

Figure 8: Full-throughput Frontier Curve. Uni-regular topologies with $\mathrm{H}=8$ and $\mathrm{H}=7$ can not scale well while preserving full throughput even though they maintain full BBW up to a very large size.

Xpander, there are no FatClique topologies above $10 \mathrm{~K}$ which have full throughput by the тUв for $H$ values of 7 and 8 (at these values, above $10 \mathrm{~K}$, all instances are labeled BBW).

Takeaways. While uni-regular topologies have elegant designs (Jellyfish and Xpander) and useful manageability properties (FatClique), their throughput scaling is fundamentally limited (§4), and many of their topology instances do not have full-throughput even at scales far smaller than modern data centers (e.g., Amazon AWS with more than $50 \mathrm{~K}$ servers [2], Google Jupiter with more than $30 \mathrm{~K}$ servers [42]). At these larger scales, these topologies can use smaller values of $H$, but this can negate the cost advantages of uni-regular topologies, as we show next.

\section{A THROUGHPUT-CENTRIC VIEW OF TOPOLOGY EVALUATIONS}

In this section, we revisit prior work on topology evaluation from a throughput-centric perspective.

\subsection{Throughput vs. Bisection Bandwidth}

$\S 4.1$ shows that, for uni-regular topologies, throughput and bisection bandwidth are different, and that, by definition, throughput accurately captures the capacity of the network. Here we explore whether conclusions from prior work that has used bisection bandwidth to evaluate uni-regular topologies would change if throughput were used instead. Table 4 summarizes our findings.

Topology Cost. Datacenter designers seek highly cost-effective designs [35]. FatClique [52] and Jellyfish [44] have compared the cost of their designs against Clos-based topologies by generating full bisection bandwidth instances of their topology using the minimum number of switches, and then comparing that number against a Clos with the same number of servers. Figure 9 shows what would happen if they had, instead, generated full throughput instances, for topologies with different sizes and switch radices.

Figure 9(a) and Figure 9(b) show that the full throughput Jellyfish and Xpander built from 32-port switches use about 33\% more switches than the full bisection bandwidth topology at the scale of $32 \mathrm{~K}$ and $131 \mathrm{~K}$ servers (because, to achieve full throughput at larger sizes, uni-regular topologies must use a smaller $H$ ). This increase in the number of switches for FatClique is approximately $27 \%$. This

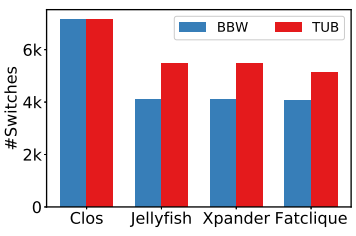

(a) $\mathrm{N}=32 \mathrm{~K}, \mathrm{R}=32$

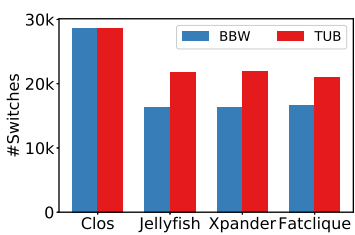

(b) $\mathrm{N}=131 \mathrm{~K}, \mathrm{R}=32$

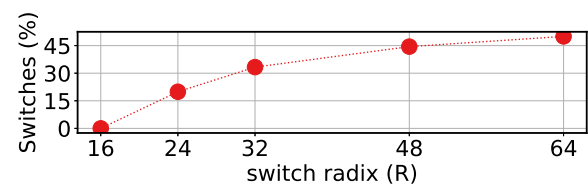

(c) Jellyfish Full Throughput vs Full BBW

FIGURE 9: Topology Cost. Number of switches to build a full throughput topology is larger than a full BBW topology. (a) Number of switches to build a topology with $32 \mathrm{~K}$ servers using 32-port switches. (b) Number of switches to build a topology with $131 \mathrm{~K}$ servers using 32 -port switches (At these scales, TUB is expected to have a small throughput gap.) (c) Number of switches to build a Jellyfish topology with different switch radices to support the same number of servers as a 1/8th 4-layer Clos. (Percentages are Full-тuB/Full-BBW - 1.)

affects the comparison with $\mathrm{Clos}^{7}$ : Clos uses 1.8x more switches compared to uni-regular topologies to achieve full bisection bandwidth ${ }^{8}$ but only $1.3 x$ more relative to full throughput uni-regular topologies.

Figure 9(c) demonstrates that, at higher switch radices, the impact of the choice of metric is more severe for uni-regular topologies. To do this experiment, we needed to normalize the scale of the topology relative to the radix of a switch. A natural way to normalize this is to design a uni-regular topology with as many servers as a full Clos with a given switch radix. However, at a radix of 64, a full Clos has 2.1M servers to which our TUB implementation does not yet scale. So, we normalize the topology scale by designing Jellyfish

\footnotetext{
${ }^{7}$ In this and subsequent evaluations, for Clos topologies the number of servers per switch for leaf switches is always equal to $\frac{R}{2}$, where $R$ is the switch radix, while the rest of the switches have no servers.

${ }^{8}$ Results for bisection bandwidth are consistent with findings of [44, 52]
} 


\begin{tabular}{|c|c|c|}
\hline \multirow{2}{*}{$\overline{\tilde{s}}$} & [52] & llyfish, Xpander, and FatClique use $\mathbf{5 0 \%}$ fewer switches to support the same servers as Clos at large-scale. \\
\hline & TUB & Jellyfish, Xpander, and FatClique use $\mathbf{2 5 \%}$ fewer switches to support the same servers as Clos at large-scale. \\
\hline \multirow{2}{*}{ 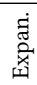 } & [44] & Jellyfish using random rewiring can be expanded with minor bandwidth loss while keeping the servers per switch constant (even under large expansion) \\
\hline & TUB & $\begin{array}{l}\text { Expanding jellyfish without considering the target size can cause significant throughput drop when servers per switch is preserved (even under small } \\
\text { expansion). }\end{array}$ \\
\hline
\end{tabular}

TABLE 4: Throughput vs. Bisection Bandwidth. Conclusions can change significantly.

topologies with the same number of servers as a $1 / 8$ th Clos for the corresponding switch radix. At a radix of 64 , a $1 / 8$ th $\mathrm{Clos}$ has $263 \mathrm{~K}$ servers. Figure 9(c) shows the percentage increase in the number of switches required to support Full Throughput over those required to support Full BBW. This fraction increases with switch radix; with 64-port switches, Full BBW requires almost 50\% more switches.

This difference can change a topology designer's tradeoff analysis. Clos and uni-regular topologies differ in one other important way: the former has demonstrated, through wide deployment, a simple and practical routing scheme (ECMP) that can achieve high throughput, but proposed routing for uni-regular topologies rely on routing schemes such as MPTCP [48] over K-shortest paths [49], ECMP-VLB hybrid [29] or FatPaths [7]. The deployment and operational cost of these schemes is not known, so, if the relative switch cost advantage of uni-regular topologies is low, a designer might find them less attractive when other costs, such as routing, are taken into account.

Fabric Expansion. As recent work has shown [52, 53], datacenter fabrics are rarely deployed at full scale initially. Rather, for a Closbased topology like Jupiter [42], a designer starts by determining a target number of servers in the datacenter and the number of layers needed in the Clos topology to achieve that scale. Then, they can incrementally deploy the topology, often in units of superblocks [53].

One attractive aspect of some uni-regular topologies like Jellyfish over Clos is that, at least conceptually, their expansion is simpler and requires no advance planning $[44,47,52]$. For example, it is possible to add one switch and its servers to Jellyfish by randomly removing links and connecting the opened ports to the new switch. It is easy to see, from Figure 8(a), that this expansion likely preserves full bandwidth. For example, if one starts with a $5 \mathrm{~K}$ Jellyfish topology with $H=8$, and augments it to $10 \mathrm{~K}$ servers, the resulting topology is still under the BBW line, so has full bisection bandwidth.

However, this expansion strategy may not always preserve full throughput. In the same example, at $10 \mathrm{~K}$ servers with $H=8$, the topology is above the Throughput line: in other words, while the topology before expansion has full throughput, the final topology does not.

Thus, when planning a datacenter topology, a designer must carefully consider future target expansion sizes and choose $\mathrm{H}$ accordingly. If the target size is $10 \mathrm{~K}$, the topology designer needs to plan in advance (as in Clos) and start with a $H=7$ instance in order to preserve throughput after expansion. (The alternative is to re-wire servers, which can significantly increase the cost of expansion).

Over-subscription. The Fat-Tree work [1] defined a topology's over-subscription ratio as the ratio between the actual bisection bandwidth and full bisection bandwidth. This definition can be misleading when applied to uni-regular topologies. For these topologies,

\begin{tabular}{|c|c|c|c|c|}
\hline Topology & $\mathrm{N}$ & $\mathrm{H}$ & $\mathrm{BBW}$ & Throughput \\
\hline Jellyfish & $32 \mathrm{~K}$ & 10 & $3: 4$ & $1: 2$ \\
Xpander & $32 \mathrm{~K}$ & 10 & $3: 4$ & $1: 2$ \\
FatClique & $32 \mathrm{~K}$ & 8.6 & $3: 4$ & $2: 3$ \\
Clos & $32 \mathrm{~K}$ & 32 & $1: 2$ & $1: 2$ \\
\hline
\end{tabular}

TABLE 5: Throughput-based vs $B B W$-based over-subscription ratio. Numbers in one row are computed on the same topology.

the throughput itself is a measure of over-subscription. A throughput of $f$ indicates that each server can send traffic at a fraction $f$ of its line rate, corresponding to an over-subscription ratio of $1: \frac{1}{f}$. Table 5 illustrates the difference between these two definitions of over-subscription ratio for uni-regular topologies. For all uniregular topologies we have measured, the over-subscription ratio defined using throughput is lower than bisection bandwidth-based over-subscription ratio. ${ }^{9}$ For Clos, these two values are identical.

This suggests that, for uni-regular topologies, throughput is a more conservative measure of over-subscription. It is also more accurate, since it measures the upper bound of the actual achievable throughput.

\subsection{Scaling Throughput Evaluations}

$\S 3$ shows that TUв better estimates worst-case throughput and scales better than most of the previous throughput estimators. Here we revisit the conclusions from prior work that has evaluated topology properties at smaller-scales using other ways to estimate throughput. Table 6 summarizes our findings; we describe these below.

Cost and Expansion. Singla et al. [44] have estimated throughput using ideal routing on a few random permutations and show that Jellyfish can support $27 \%$ more servers at full throughput than a Fat-Tree [1] using the same number of switches. They conjecture that this advantage improves by using a higher radix switch. In $\S \mathrm{K}$, we show that: (1) the cost advantage at the largest considered size in [44] is only $8 \%$ when TUB is used to estimate throughput, and (2) the cost advantage does not improve by using a higher radix switch. Similarly, Xpander has used ideal routing on all-to-all traffic matrices to estimate the throughput, and has shown that their topology is more cost efficient than Fat-tree, and allows incremental expandability up to any size with minor throughput loss. In $\S \mathrm{L}$, we show that throughput of Xpander can drop significantly when

\footnotetext{
${ }^{9}$ The instance of FatClique we chose for this experiment uses a different $H$ than the instances of Jellyfish and Xpander, which is why it has a different throughput.
} 


\begin{tabular}{|c|c|c|}
\hline \multirow{4}{*}{ 范 } & [44] & $\begin{array}{l}\text { Jellyfish supports } 27 \% \text { more servers at full throughput than a (same-equipment) Fat-tree (at }<900 \text { servers) and this advantage improves by using higher port } \\
\text { switches. }\end{array}$ \\
\hline & TUB & Jellyfish supports $\mathbf{8 \%}$ more servers at considered size and the advantage does not always improve by using higher port switches. \\
\hline & [47] & Xpander uses $80 \%-\mathbf{8 5 \%}$ switches to support the same number of servers as Fat-tree at the scale of $<4 \mathrm{~K}$ servers. \\
\hline & TUB & $\begin{array}{l}\text { At the largest considered size in [47], Xpander uses more than 95\% switches. However, at larger scale (>40K servers), Xpander uses 80\% switches (matching } \\
\text { the number reported in [47]) }\end{array}$ \\
\hline \multirow{2}{*}{ 家 } & [47] & Xpander using random rewiring can be incrementally expanded to any size while preserving high performance. \\
\hline & TUB & Expanding Xpander without considering the target size can cause significant throughput drop, leading to a topology with less than full-throughput. \\
\hline \multirow{4}{*}{ 节 } & [44] & Jellyfish is highly resilient to random link failures at the scale of <1K servers built using 12-port switches. \\
\hline & TUB & At some scales, Jellyfish can be as much as $20 \%$ less resilient compared to optimal resiliency using 32-port switches.. \\
\hline & [47] & Xpander is resilient to failures at the scale of $<\mathbf{1 K}$ servers built using 14-port switches. \\
\hline & TUB & At some scales, Xpander can be as much as $\mathbf{2 0 \%}$ less resilient compared to optimal resiliency using 32-port switches. \\
\hline
\end{tabular}

TAвLE 6: Scaling Throughput Evaluations. Conclusions can change significantly.

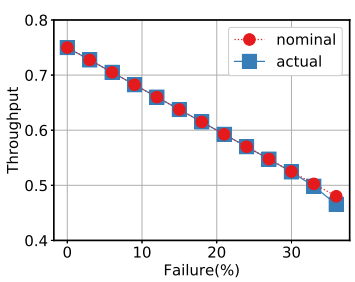

(a) $\mathrm{N}=32 \mathrm{~K}$

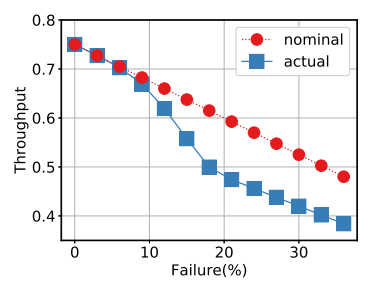

(b) $\mathrm{N}=131 \mathrm{~K}$

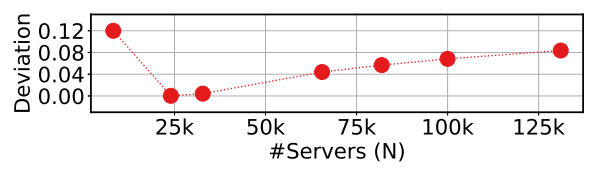

(c) Variation

FIGURE 10: Throughput of uni-regular topologies under random link failure. Large uni-regular topologies degrade less than gracefully with failure.

using random rewiring even for very small expansions, resulting in a topology with less than full-throughput (similar to Jellyfish).

Failure Resiliency. Prior work has explored the resilience of Jellyfish [44] and Xpander [47] to random link failures for relatively small topologies (at the scale of a few thousand servers). To do this, they compute the throughput achieved by ideal routing (using multi-commodity flow, which limits scaling) for a few randomly chosen permutation matrices. The showed that, at these scales, these topologies degrade gracefully, defined as follows. If $\theta$ is the throughput of a topology without failure, and a randomly chosen fraction $f$ of all links fail, then the nominal throughput under failure is $(1-f) \theta$ (other work [45] has used a similar definition to assess failure resilience in WAN switches). We say a topology degrades gracefully if the actual throughput (in our experiments, the throughput upper bound) under failure closely matches the nominal throughput under failure.

TUB allows us to evaluate failure resilience of these topologies at larger scales.

Figure 10 shows the throughput behavior of Jellyfish with 8 servers per switch under random link failures, based on TUB for: (a) $32 \mathrm{~K}$, (b) $131 \mathrm{~K}$. Jellyfish with $32 \mathrm{~K}$ servers is perfectly resilient for up to $30 \%$ link failure and deviates by $<1 \%$ afterward while $131 \mathrm{~K}$ server topology is perfectly resilient for up to $11 \%$ link failures and then deviates by $20 \%$ from the nominal throughput. This deviation occurs because, the $131 \mathrm{~K}$ topology has a relatively smaller number of shortest paths (compared to the $32 \mathrm{~K}$ topology) between each pair in the maximal permutation matrix (Figure 4(b)). Higher rates of random failures can reduce the available shortest paths even further, reducing throughput.

This relationship between deviation from the nominal, and the number of shortest paths, is more evident when comparing Figure 10(c) with Figure 4(b). The former plots the root mean square deviation from the nominal as a function of topology size. In the latter, the number of shortest paths decreases steadily from $24 \mathrm{~K}$ to $131 \mathrm{~K}$; in Figure 10(c), the deviation increases correspondingly. Xpander exhibits same behavior as Jellyfish under random link failures.

Takeaway. This example illustrates how TUB can reveal previously unobserved properties of a topology at larger scales. Using our bound, we are able to measure the resiliency of uni-regular topologies for up to $131 \mathrm{~K}$. Using the throughput estimators in [44, 47] (full-blown MCF), we are unable to scale beyond $8 \mathrm{~K}$ servers on our platform.

\section{PRACTICAL CONSIDERATIONS}

The importance of worst-case bounds. Focusing on worst-case bounds can result in pessimistic designs and evaluations. In many situations, it may be appropriate to focus on average case performance. However, datacenter topologies, once deployed, are used for several years [42]; in this time, traffic demands can grow significantly. Because it is hard to predict demand over longer time-frames, datacenter designers have focused on worst-case measures (like bisection bandwidth) as a design aid to maximize the lifetime of their designs. тUB follows this line of thinking: this paper shows that TUB is a better measure of worst-case performance for uni-regular topologies than bisection bandwidth.

Clos-based deployments. Most deployed datacenter designs today are Clos-based. However, designers are actively exploring other lower-cost designs, one of which is the spine-free design [22], in which the spine or topmost layer of switch blocks is replaced by direct connections between the intermediate-layer (or aggregation layer) pods [1]. Pods may carry transit traffic between other pods. In this design, the inter-pod topology is effectively uni-regular, for which TUB can be used to understand performance.

Practical Workloads. In this paper, we have compared fullbisection bandwidth topologies with full throughput topologies. 
Deployed topologies are often over-subscribed; a deployed Clos might have less than full bisection bandwidth. These deployments work well because operators carefully manage datacenter workloads to ensure that they don't exceed fabric capacity. They also leave spare capacity for management operations such as expansion and upgrade [42, 53]. For Clos, the bisection bandwidth of the oversubscribed topology is a good measure of the capacity. For uni-regular topologies, TUB is a better measure of capacity for an oversubscribed network (§5.1).

Benchmarking routing designs. Aside from topology, routing design also determines whether the datacenter is able effectively utilize its capacity in serving workloads. For uni-regular topologies, or variants thereof, TUB can be used to understand how well a proposed routing design can utilize capacity.

\section{RELATED WORK}

Datacenter Designs. Prior work has investigated a large body of topology designs focusing on high bisection bandwidth, costeffective topologies with low diameter [8, 30, 44, 47, 52]. Our paper addresses the performance of many of these topology designs. We do not evaluate topologies such as SlimFly [6] and Dragonfly [30]. These focus on reducing latency, but, to scale to today's datacenters, they generally need switches with much higher port counts than available with merchant silicon. For instance, with a 64-port switch, a SlimFly can support $32 \mathrm{~K}$ servers, but a 4-stage Clos can accommodate $2.1 \mathrm{M}$. We emphasize that TUB applies to these two topologies as well as they are uni-regular. Prior work has described server-centric topologies such as DCell [17] and BCube [16] which equip servers with multiple ports and route packets through servers. Server-based forwarding can be highly unreliable [42], so deployed datacenters have not adopted these designs, and we have not considered these in this paper. Future work can explore throughput bounds for this class of topologies.

A more recent direction focuses on reconfigurable topology designs $[13,14,20,37,38,54]$ that adapt the topology in response to the observed traffic. Most reconfigurable topology designs adapt instantaneously to shifts in traffic demand, and attempt to minimize flow completion times. To the extent that each adapted topology is uni-regular or bi-regular, Theorem 2.2 will apply to the topology. However, we have left it to future work to understand how topology throughput relates to the objective of minimizing flow completion times, the focus of topology reconfiguration.

Throughput. As discussed earlier, significant prior work exists on throughput in datacenters. Some work [50] has explored the application-level throughput under different traffic conditions. Prior work has developed a theoretical understanding of throughput [12, 27, 43]. Of these, [12] compares performance of 3 throughputapproximating algorithms (Jain [24], Hoefler [23, 51], and an LPbased approximation), and show that Jain method is a more accurate approximation model compared to the other two in capturing the average throughput over all the flows. More recently, [43] focuses on approximating average throughput under uniform traffic, and [27] studies the relationship between traffic-dependent sparsestcut and throughput at the scale of few thousand servers. Inspired especially by the latter two papers, we derive a tight throughput upper bound across all traffic matrices and explore it to understand practical scaling limits for uni-regular topologies, and the utility of a throughput-centric view in evaluating properties of datacenter topologies. We also compare тUв against many of these prior approaches.

Practical Routing. In practice, throughput highly depends on the routing algorithm and the underlying topology. ECMP is optimal for the Clos family $[1,15,42]$. For Jellyfish, Xpander, and FatClique, routing strategies like an ECMP-VLB hybrid [29] and FatPaths [7] have shown promising throughput performance. We have left it to future work to understand the gap between achievable throughput using these more practical routing strategies and тUв.

\section{CONCLUSIONS AND FUTURE WORK}

This paper broadens our understanding of the throughput metric for datacenter topology performance, and its relationship to bisection bandwidth. We derive a closed-form expression for the upper bound of the throughput (TUB) of a given topology that is independent of routing. This bound applies to most proposed datacenter topologies. For a sub-class of these designs, uni-regular topologies, we are able to derive an upper-bound on throughput that applies to any instance in this sub-class, using which we show that uni-regular topologies are fundamentally limited: beyond a certain scale, they cannot have full throughput even if they have full bisection bandwidth. In practice, many instances of uni-regular topologies with $10-15 \mathrm{~K}$ servers cannot have full throughput. Finally, we demonstrate that TUB to evaluate properties of a topology can result in different conclusions compared to using other metrics. Future work can explore the throughput gap between TUB and the throughput achievable using practical routing algorithms, explore the throughput of Clos-variants like [36], scale TUB to even larger topologies, and improve its tightness.

Acknowledgements. We thank our shepherd Michael Schapira, and the anonymous reviewers for their feedback on the paper. This material is based upon work supported by the U.S. National Science Foundation under grants No. CNS-1901523, CNS-1705086, and CNS1955422 . 


\section{REFERENCES}

[1] Mohammad Al-Fares, Alexander Loukissas, and Amin Vahdat. 2008. A Scalable, Commodity Data Center Network Architecture. In Proceedings of the ACM SIGCOMM 2008 Conference on Data Communication (SIGCOMM '08). Association for Computing Machinery, New York, NY, USA, 63-74. https://doi.org/10.1145/ 1402958.1402967

[2] Shahbaz Alam, Pawan Agnihotri, and Greg Dumont. 2016. AWSre:Invent. Enterprise fundamentals: design your account and VPC architecture for enterprise operating models. https://www.slideshare.net/AmazonWebServices/aws-reinvent2016-enterprise-fundamentals-design-your-account-and-vpc-architecture-forenterprise-operating-models-ent203. (2016)

[3] Alexey Andreyev. 2014. Introducing Data Center Fabric, the Next-generation Facebook Data Center Network. https://engineering.fb.com/productionengineering/introducing-data-center-fabric-the-next-generation-facebookdata-center-network/. (2014).

[4] Jordi Arjona Aroca and Antonio Fernández Anta. 2014. Bisection (Band)Width of Product Networks with Application to Data Centers. IEEE Transactions on Parallel and Distributed Systems 25, 3 (2014), 570-580. https://doi.org/10.1109/ TPDS.2013.95

[5] Dimitri P. Bertsekas, Angelia Nedić, and Asuman E. Ozdaglar. 2003. Convex Analysis and Optimization. Athena Scientific.

[6] Maciej Besta and Torsten Hoefler. 2014. Slim Fly: A Cost Effective Low-Diameter Network Topology. In Proceedings of the International Conference for High Perfor mance Computing, Networking, Storage and Analysis (SC '14). IEEE Press, 348-359. https://doi.org/10.1109/SC.2014.34

[7] Maciej Besta, Marcel Schneider, Karolina W Cynk, Marek Konieczny, Erik Henriksson, Salvatore Di Girolamo, Ankit Singla, and Torsten Hoefler. 2019. FatPaths: Routing in Supercomputers, Data Centers, and Clouds with Low-Diameter Networks When Shortest Paths Fall Short. ArXiv abs/1906.10885 (2019).

[8] C. Clos. 1953. A Study of Non-blocking Switching Networks. The Bell System Technical fournal 32, 2 (Mar. 1953)

[9] Gabor Csardi and Tamas Nepusz. 2006. The Igraph Software Package for Complex Network Research. Interfournal Complex Systems (2006), 1695. http://igraph.org

[10] Andrew R. Curtis, Tommy Carpenter, Mustafa Elsheikh, Alejandro López-Ortiz, and S. Keshav. 2012. REWIRE: An optimization-based framework for unstructured data center network design. In 2012 Proceedings IEEE INFOCOM. 1116-1124. https://doi.org/10.1109/INFCOM.2012.6195470

[11] N. G. Duffield, Pawan Goyal, Albert Greenberg, Partho Mishra, K. K. Ramakrishnan, and Jacobus E. van der Merive. 1999. A Flexible Model for Resource Management in Virtual Private Networks. SIGCOMM Comput. Commun. Rev. 29, 4 (Aug. 1999), 95-108. https://doi.org/10.1145/316194.316209

[12] Peyman Faizian, Md Atiqul Mollah, Md Shafayat Rahman, Xin Yuan, Scott Pakin, and Mike Lang. 2017. Throughput Models of Interconnection Networks: The Good, the Bad, and the Ugly. In 2017 IEEE 25th Annual Symposium on HighPerformance Interconnects (HOTI). 33-40.

[13] Nathan Farrington, George Porter, Sivasankar Radhakrishnan, Hamid Hajabdolal Bazzaz, Vikram Subramanya, Yeshaiahu Fainman, George Papen, and Amin Vahdat. 2010. Helios: A Hybrid Electrical/Optical Switch Architecture for Modular Data Centers. In Proceedings of the ACM SIGCOMM 2010 Conference (SIGCOMM '10). Association for Computing Machinery, New York, NY, USA, 339-350. https: //doi.org/10.1145/1851182.1851223

[14] Monia Ghobadi, Ratul Mahajan, Amar Phanishayee, Nikhil Devanur, Janardhan Kulkarni, Gireeja Ranade, Pierre-Alexandre Blanche, Houman Rastegarfar, Madeleine Glick, and Daniel Kilper. 2016. ProjecToR: Agile Reconfigurable Data Center Interconnect. In Proceedings of the 2016 ACM SIGCOMM Conference (SIGCOMM '16). Association for Computing Machinery, New York, NY, USA, 216-229. https://doi.org/10.1145/2934872.2934911

[15] Albert Greenberg, James R. Hamilton, Navendu Jain, Srikanth Kandula, Changhoon Kim, Parantap Lahiri, David A. Maltz, Parveen Patel, and Sudipta Sengupta. 2009. VL2: A Scalable and Flexible Data Center Network. In Proceedings of the ACM SIGCOMM 2009 Conference on Data Communication (SIG COMM '09). Association for Computing Machinery, New York, NY, USA, 51-62. https://doi.org/10.1145/1592568.1592576

[16] Chuanxiong Guo, Guohan Lu, Dan Li, Haitao Wu, Xuan Zhang, Yunfeng Shi, Chen Tian, Yongguang Zhang, and Songwu Lu. 2009. BCube: A High Performance, Server-Centric Network Architecture for Modular Data Centers. In Proceedings of the ACM SIGCOMM 2009 Conference on Data Communication (SIGCOMM '09). Association for Computing Machinery, New York, NY, USA, 63-74. https: //doi.org/10.1145/1592568.1592577

[17] Chuanxiong Guo, Haitao Wu, Kun Tan, Lei Shi, Yongguang Zhang, and Songwu Lu. 2008. Dcell: A Scalable and Fault-Tolerant Network Structure for Data Centers. Proceedings of the ACM SIGCOMM 2008 conference on Data communication 38, 4 (Aug. 2008), 75-86. https://doi.org/10.1145/1402946.1402968

[18] LLC Gurobi Optimization. 2020. Gurobi Optimizer Reference Manual. (2020) http://www.gurobi.com

[19] Aric A. Hagberg, Daniel A. Schult, and Pieter J. Swart. 2008. Exploring Network Structure, Dynamics, and Function using NetworkX. In Proceedings of the 7th
Python in Science Conference, Gaël Varoquaux, Travis Vaught, and Jarrod Millman (Eds.). Pasadena, CA USA, $11-15$

[20] Navid Hamedazimi, Zafar Qazi, Himanshu Gupta, Vyas Sekar, Samir R. Das, Jon P. Longtin, Himanshu Shah, and Ashish Tanwer. 2014. FireFly: A Reconfigurable Wireless Data Center Fabric Using Free-Space Optics. In Proceedings of the 2014 ACM Conference on SIGCOMM (SIGCOMM '14). Association for Computing Machinery, New York, NY, USA, 319-330. https://doi.org/10.1145/2619239.2626328

[21] James Hamilton. 2010. Datacenter Networks are in my Way. http://goo.gl/Ho6mA. (2010).

[22] Vipul Harsh, Sangeetha Abdu Jyothi, and P. Brighten Godfrey. 2020. Spineless Data Centers. In Proceedings of the 19th ACM Workshop on Hot Topics in Networks (HotNets '20). Association for Computing Machinery, New York, NY, USA, 67-73. https://doi.org/10.1145/3422604.3425945

[23] Torsten Hoefler, Timo Schneider, and Andrew Lumsdaine. 2008. Multistage switches are not crossbars: Effects of static routing in high-performance networks. In 2008 IEEE International Conference on Cluster Computing. 116-125.

[24] Nikhil Jain, Abhinav Bhatele, Xiang Ni, Nicholas J. Wright, and Laxmikant V. Kale. 2014. Maximizing Throughput on a Dragonfly Network. In SC '14: Proceedings of the International Conference for High Performance Computing, Networking, Storage and Analysis. 336-347.

[25] A. Jajszczyk. 2003. Nonblocking, Repackable, and Rearrangeable Clos Networks: Fifty Years of the Theory Evolution. IEEE Communications Magazine 41, 10 (2003), $28-33$

[26] Sangeetha Abdu Jyothi, Ankit Singla, P Godfrey, and Alexandra Kolla. 2014. Measuring and Understanding Throughput of Network Topologies. arXiv preprint arXiv:1402.2531 (2014)

[27] Sangeetha Abdu Jyothi, Ankit Singla, P. Brighten Godfrey, and Alexandra Kolla. 2016. Measuring and Understanding Throughput of Network Topologies. In Proceedings of the International Conference for High Performance Computing, Networking, Storage and Analysis (SC '16). IEEE Press, Article 65, 12 pages.

[28] George Karypis and Vipin Kumar. 1998. A Fast and High Quality Multilevel Scheme for Partitioning Irregular Graphs. SIAM J. Sci. Comput. 20, 1 (Dec. 1998), 359-392.

[29] Simon Kassing, Asaf Valadarsky, Gal Shahaf, Michael Schapira, and Ankit Singla. 2017. Beyond Fat-Trees Without Antennae, Mirrors, and Disco-Balls. In Proceedings of the Conference of the ACM Special Interest Group on Data Communication (SIGCOMM '17). Association for Computing Machinery, New York, NY, USA, 281-294. https://doi.org/10.1145/3098822.3098836

[30] John Kim, Wiliam J. Dally, Steve Scott, and Dennis Abts. 2008. TechnologyDriven, Highly-Scalable Dragonfly Topology. In Proceedings of the 35th Annual International Symposium on Computer Architecture (ISCA '08). IEEE Computer Society, USA, 77-88. https://doi.org/10.1109/ISCA.2008.19

[31] Murali Kodialam, T. V. Lakshman, and Sudipta Sengupta. 2011. Traffic-Oblivious Routing in the Hose Model. IEEE/ACM Trans. Netw. 19, 3 (June 2011), 774-787. https://doi.org/10.1109/TNET.2010.2099666

[32] Harold W Kuhn. 1955. The Hungarian method for the assignment problem. Naval research logistics quarterly 2, 1-2 (1955), 83-97.

[33] Praveen Kumar, Yang Yuan, Chris Yu, Nate Foster, Robert Kleinberg, Petr Lapukhov, Chiun Lin Lim, and Robert Soulé. 2018. Semi-Oblivious Traffic Engineering: The Road Not Taken. In 15th USENIX Symposium on Networked Systems Design and Implementation (NSDI 18). USENIX Association, Renton, WA, 157-170. https://www.usenix.org/conference/nsdi18/presentation/kumar

[34] Tom Leighton and Satish Rao. 1999. Multicommodity Max-Flow Min-Cut Theorems and Their Use in Designing Approximation Algorithms. f. ACM 46, 6 (Nov. 1999), 787-832. https://doi.org/10.1145/331524.331526

[35] Hong Liu, Ryohei Urata, Xiang Zhou, and Amin Vahdat. 2020. Evolving Requirements and Trends of Datacenters Networks. Springer International Publishing, Cham, 707-724. https://doi.org/10.1007/978-3-030-16250-4_21

[36] Vincent Liu, Daniel Halperin, Arvind Krishnamurthy, and Thomas Anderson. 2013. F10: A Fault-Tolerant Engineered Network. In Proceedings of the 10th USENIX Conference on Networked Systems Design and Implementation (NSDI'13). USENIX Association, USA, 399-412.

[37] William M. Mellette, Rajdeep Das, Yibo Guo, Rob McGuinness, Alex C. Snoeren, and George Porter. 2020. Expanding Across Time to Deliver Bandwidth Efficiency and Low Latency. In 17th USENIX Symposium on Networked Systems Design and Implementation (NSDI 20). USENIX Association, Santa Clara, CA, 1-18. https: //www.usenix.org/conference/nsdi20/presentation/mellette

[38] William M. Mellette, Rob McGuinness, Arjun Roy, Alex Forencich, George Papen, Alex C. Snoeren, and George Porter. 2017. RotorNet: A Scalable, LowComplexity, Optical Datacenter Network. In Proceedings of the Conference of the ACM Special Interest Group on Data Communication (SIGCOMM '17). Association for Computing Machinery, New York, NY, USA, 267-280. https: //doi.org/10.1145/3098822.3098838

[39] Mirka Miller and Jozef vSirávn. 2005. Moore Graphs and Beyond: A Survey of the Degree/diameter Problem. Electronic fournal of Combinatorics, Dynamic survey 14 (12 2005), 1-61.

[40] James Munkres. 1957. Algorithms for the assignment and transportation problems. Fournal of the society for industrial and applied mathematics 5, 1 (1957), 
32-38.

[41] S. Ohta. 1987. A Simple Control Algorithm for Rearrangeable Switching Networks with Time Division Multiplexed Links. IEEE fournal on Selected Areas in Communications 5, 8 (1987), 1302-1308.

[42] Arjun Singh, Joon Ong, Amit Agarwal, Glen Anderson, Ashby Armistead, Roy Bannon, Seb Boving, Gaurav Desai, Bob Felderman, Paulie Germano, Anand Kanagala, Jeff Provost, Jason Simmons, Eiichi Tanda, Jim Wanderer, Urs Hölzle, Stephen Stuart, and Amin Vahdat. 2015. Jupiter Rising: A Decade of Clos Topologies and Centralized Control in Google's Datacenter Network. In Proceedings of the 2015 ACM Conference on Special Interest Group on Data Communication (SIGCOMM '15). Association for Computing Machinery, New York, NY, USA, 183-197. https://doi.org/10.1145/2785956.2787508

[43] Ankit Singla, P. Brighten Godfrey, and Alexandra Kolla. 2014. High Throughput Data Center Topology Design. In Proceedings of the 11th USENIX Conference on Networked Systems Design and Implementation (NSDI'14). USENIX Association, USA, $29-41$.

[44] Ankit Singla, Chi-Yao Hong, Lucian Popa, and P. Brighten Godfrey. 2012. Jellyfish: Networking Data Centers Randomly. In 9th USENIX Symposium on Networked Systems Design and Implementation (NSDI 12). USENIX, San Jose, CA, 225-238. https: //www.usenix.org/conference/nsdi12/technical-sessions/presentation/singla

[45] Sucha Supittayapornpong, Barath Raghavan, and Ramesh Govindan. 2019. Towards Highly Available Clos-Based WAN Routers. In Proceedings of the ACM Special Interest Group on Data Communication (SIGCOMM '19). Association for Computing Machinery, New York, NY, USA, 424-440. https://doi.org/10.1145/ 3341302.3342086

[46] Brian Towles and William J. Dally. 2002. Worst-Case Traffic for Oblivious Routing Functions. In Proceedings of the Fourteenth Annual ACM Symposium on Parallel Algorithms and Architectures (SPAA '02). Association for Computing Machinery, New York, NY, USA, 1-8. https://doi.org/10.1145/564870.564872

[47] Asaf Valadarsky, Gal Shahaf, Michael Dinitz, and Michael Schapira. 2016. Xpander: Towards Optimal-Performance Datacenters. In Proceedings of the 12th International on Conference on Emerging Networking EXperiments and Technologies (CoNEXT '16). Association for Computing Machinery, New York, NY, USA, 205-219. https://doi.org/10.1145/2999572.2999580

[48] Damon Wischik, Costin Raiciu, Adam Greenhalgh, and Mark Handley. 2011 Design, Implementation and Evaluation of Congestion Control for Multipath TCP. In Proceedings of the 8th USENIX Conference on Networked Systems Design and Implementation (NSDI'11). USENIX Association, USA, 99-112.

[49] Jin Y. Yen. 1971. Finding the K Shortest Loopless Paths in a Network. Management Science 17, 11 (1971), 712-716. http://www.jstor.org/stable/2629312

[50] Xin Yuan, Santosh Mahapatra, Michael Lang, and Scott Pakin. 2014. LFTI: A New Performance Metric for Assessing Interconnect Designs for Extreme-Scale HPC Systems. In IEEE 28th International Parallel and Distributed Processing Symposium. 273-282.

[51] Xin Yuan, Santosh Mahapatra, Wickus Nienaber, Scott Pakin, and Michael Lang. 2013. A New Routing Scheme for Jellyfish and Its Performance with HPC Workloads. In Proceedings of the International Conference on High Performance Computing, Networking, Storage and Analysis (SC '13). Association for Computing Machinery, New York, NY, USA, Article 36, 11 pages. https: //doi.org/10.1145/2503210.2503229

[52] Mingyang Zhang, Radhika Niranjan Mysore, Sucha Supittayapornpong, and Ramesh Govindan. 2019. Understanding Lifecycle Management Complexity of Datacenter Topologies. In 16th USENIX Symposium on Networked Systems Design and Implementation (NSDI 19). USENIX Association, Boston, MA, 235-254. https://www.usenix.org/conference/nsdi19/presentation/zhang

[53] Shizhen Zhao, Rui Wang, Junlan Zhou, Joon Ong, Jeffrey C. Mogul, and Amin Vahdat. 2019. Minimal Rewiring: Efficient Live Expansion for Clos Data Center Networks. In Proc. USENIX NSDI.

[54] Xia Zhou, Zengbin Zhang, Yibo Zhu, Yubo Li, Saipriya Kumar, Amin Vahdat, Ben Y. Zhao, and Haitao Zheng. 2012. Mirror Mirror on the Ceiling: Flexible Wireless Links for Data Centers. In Proceedings of the ACM SIGCOMM 2012 Conference on Applications, Technologies, Architectures, and Protocols for Computer Communication (SIGCOMM '12). Association for Computing Machinery, New York, NY, USA, 443-454. https://doi.org/10.1145/2342356.2342440 


\section{APPENDIX}

Appendices are supporting material that have not been peerreviewed.

\section{A Proof of Theorem 2.1}

Proof. In a doubly-stochastic matrix, each row and each column contain non-negative values that add up to 1 . The Birkhoff-von Neumann theorem states that the $n \times n$ permutation matrices form the vertices of the convex polytope containing the set of $n \times n$ doubly-stochastic matrices. We observe that $\mathcal{T}$ contains all doublystochastic matrices scaled by $H$. From the Birkhoff-von Neumann theorem, it follows that the vertices of the convex polytope containing $\mathcal{T}$ is the set of traffic matrices in $\hat{\mathcal{T}}$. It remains to show that the minimum throughput across $\hat{\mathcal{T}}$ is always equal to that across $\mathcal{T}$.

To prove that $\min _{T \in \mathcal{T}} \theta(T)=\min _{T \in \hat{\mathcal{T}}} \theta(T)$, let $\theta^{*}=\theta\left(T^{*}\right)$ be the minimum of the LHS achieved at traffic matrix $T^{*} \in \mathcal{T}$. We will show by contradiction that at least one permutation traffic $T \in \hat{\mathcal{T}}$ leads to this $\theta^{*}$. Specifically, let $\theta^{*}=\min _{T \in \hat{\mathcal{T}}} \theta(T)$. Suppose there is no such permutation traffic matrix. Let $\hat{\theta}>\theta^{*}$ and $\hat{\theta}=\min _{T \in \hat{\mathcal{T}}} \theta(T)$ be the minimum achieved by some permutation traffic matrix in $\hat{\mathcal{T}}$. Caratheodory's theorem [5] implies that there exists at most $|\mathcal{K}|^{2}+1$ permutation traffic matrices $\left\{T_{x}\right\}$ in $\hat{\mathcal{T}}$ such that

$$
T^{*}=\sum_{x=1}^{|\mathcal{K}|^{2}+1} \lambda_{x} T_{x}, \quad \sum_{x=1}^{|\mathcal{K}|^{2}+1} \lambda_{x}=1, \text { and } \lambda_{x} \in[0,1] \quad \forall x .
$$

Given this, we can use a convex combination of permutation traffic matrices $\left\{T_{x}\right\}$ and $\left\{\lambda_{x}\right\}$ to construct traffic matrix $T^{*}$ and a solution to the multi-commodity flow problem under $T^{*}$. The throughput of this solution cannot be less than $\hat{\theta}$, since all permutation traffic matrices have a throughput of at least $\hat{\theta}$. This leads to a contradiction, because we have assumed that $\theta^{*}<\hat{\theta}$. Thus, there must exist a permutation traffic matrix $T_{x} \in \hat{\mathcal{T}}$ such that $\theta^{*}=\theta\left(T_{x}\right)$

\section{B Proof of Throughput Bound for uni-regular Topology}

Proof. Let $\mathcal{K}$ denote the set of all switches with $H$ servers. Fix a permutation traffic matrix $T$ from $\hat{\mathcal{T}}$. We solve a path-based multi-commodity flow problem $(\S \mathrm{H}$, commonly used in wide-area network traffic engineering [33]) that maximizes throughput $\theta(T)$ under this traffic matrix $T$. At each switch $u$, the ingress traffic consists of 1) traffic destined to servers attached to $u$ and 2) transit traffic $X_{u}(T)$. This ingress traffic is bounded by the capacity of network-facing ports, so we have $X_{u}(T)+\theta(T) \sum_{v \in \mathcal{K} \backslash\{u\}} t_{v u} \leq$ $R_{u}-H$ for every $u \in \mathcal{K}$, where $R_{u}$ is the number of used ports in switch $u$. (This models the fact that, for many uni-regular topologies, some ports are left unused on switches.) Summing over $u \in \mathcal{K}$ gives

$$
\sum_{u \in \mathcal{K}} X_{u}(T) \leq \sum_{u \in \mathcal{K}}\left(R_{u}-H\right)-\theta(T) \sum_{u \in \mathcal{K}} \sum_{v \in \mathcal{K} \backslash\{u\}} t_{v u} .
$$

The LHS of the above inequality is equal to the total transit traffic in the network caused by traffic matrix $T$. Alternatively, we can compute the total transit traffic based on the set of paths $\mathcal{P}_{u v}$ and split ratios for those paths $\left\{\beta_{p}(T)\right\}$ as

$$
\sum_{u \in \mathcal{K}} X_{u}(T)=\theta(T) \sum_{u \in \mathcal{K}} \sum_{v \in \mathcal{K} \backslash\{u\}} t_{u v} \sum_{p \in \mathcal{P}_{u v}} \beta_{p}(T)(\operatorname{len}(p)-1) .
$$

Since all the paths in $\mathcal{P}_{u v}$ are at least the shortest path and $\sum_{p \in \mathcal{P}_{u v}} \beta_{p}(T)=1$ for all $u, v \in \mathcal{K}^{2}$, we can rewrite the above equation as an inequality:

$$
\sum_{u \in \mathcal{K}} X_{u}(T) \geq \theta(T) \sum_{u \in \mathcal{K}} \sum_{v \in \mathcal{K} \backslash\{u\}} t_{u v}\left(L_{u v}-1\right) .
$$

From Equation 4 and Equation 6, we have

$$
\theta(T) \leq \frac{\sum_{u \in \mathcal{K}}\left(R_{u}-H\right)}{\sum_{u \in \mathcal{K}} \sum_{v \in \mathcal{K} \backslash\{u\}} t_{u v} L_{u v}} .
$$

This throughput holds under every traffic matrix $T$ for every $T \in \hat{\mathcal{T}}$. Taking the minimum over the set yields

$$
\theta^{*}=\min _{T \in \hat{\mathcal{T}}} \theta(T) \leq \min _{T \in \hat{\mathcal{T}}} \frac{\sum_{u \in \mathcal{K}}\left(R_{u}-H\right)}{\sum_{u \in \mathcal{K}} \sum_{v \in \mathcal{K} \backslash\{u\}} t_{u v} L_{u v}} .
$$

Finally, using the facts that (a) $\sum_{u \in \mathcal{K}}\left(R_{u}-H\right)=2 E$, (b) every traffic matrix is a permutation traffic, and (c) the length of the shortest path from a switch to itself is equal to 0 , we have the throughput upper bound in Equation 1.

\section{Proof of Throughput Bound for bi-regular Topology}

Proof. Let $\mathcal{S}$ and $\mathcal{K}$ denote the set of all switches and switches with $H$ servers respectively. Fix a permutation traffic matrix $T$ from $\hat{\mathcal{T}}$. We solve a path-based multi-commodity flow problem that maximizes throughput $\theta(T)$ under this traffic matrix $T$. At each switch $u$, the ingress traffic consists of 1) traffic destined to servers attached to $u$ and 2) transit traffic $X_{u}(T)$. This ingress traffic is bounded by the capacity of network-facing ports, and we have $X_{u}(T)+\theta(T) \sum_{v \in \mathcal{K} \backslash\{u\}} t_{v u} \leq R_{u}-H_{u}$ for every $u \in \mathcal{K}$. Summing over $u \in \mathcal{K}$ gives

$$
\sum_{u \in \mathcal{K}} X_{u}(T) \leq \sum_{u \in \mathcal{K}}\left(R_{u}-H_{u}\right)-\theta(T) \sum_{u \in \mathcal{K}} \sum_{v \in \mathcal{K} \backslash\{u\}} t_{v u} .
$$

Similarly, at every switch $u$ with no directly connected server, the ingress traffic only consists of transit traffic $X_{u}(T)$, and we have $X_{u}(T) \leq R_{u}-H_{\mathcal{u}}$ for every $u \in \mathcal{S} \backslash \mathcal{K}$. Summing over $u \in \mathcal{S} \backslash \mathcal{K}$ gives

$$
\sum_{u \in \mathcal{S} \backslash \mathcal{K}} X_{\mathcal{u}}(T) \leq \sum_{u \in \mathcal{S} \backslash \mathcal{K}}\left(R_{\mathcal{u}}-H_{u}\right) .
$$

From Equation 7 and Equation 8, we have

$$
\sum_{u \in \mathcal{S}} X_{u}(T) \leq \sum_{u \in \mathcal{S}}\left(R_{u}-H_{u}\right)-\theta(T) \sum_{u \in \mathcal{K}} \sum_{v \in \mathcal{K} \backslash\{u\}} t_{v u}
$$

The rest of the proof is similar to Theorem $2.2(\S \mathrm{B})$. 


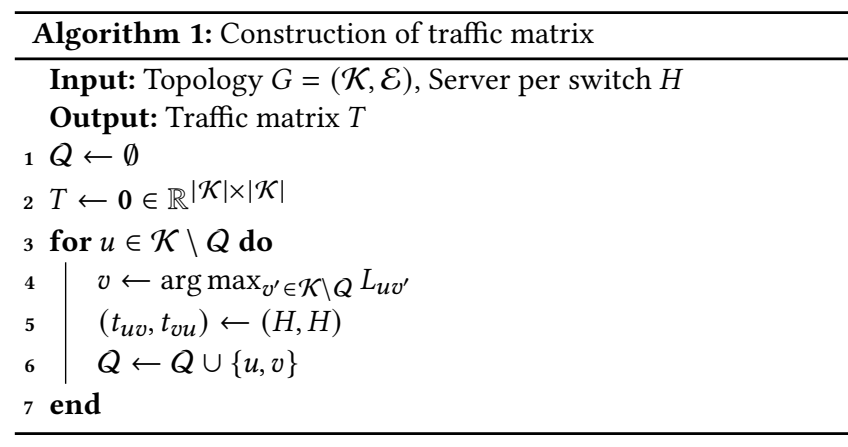

\section{Proof of Theorem 4.1.}

Lemma 8.1. Given a uni-regular topology with total servers $N$ and $H$ servers per switch, for every switch $u$, the number of switches with at least $m$ hops away from the switch is at least

$$
W_{m}=\frac{N}{H}-1-(R-H) \frac{(R-H-1)^{m-1}-1}{R-H-2}, \quad m \in\{1, \ldots, d\}
$$

where $d$ is the minimum diameter computed using Moore bound [39].

Proof. Fix switch $u$. Let $y_{i}$ be the number of switches with distance $i$ from switch $u$. Since every switch has $R-H$ switchto-switch ports, the number of switches with distance 1 from $u$ is bouned by $y_{1} \leq R-H$. The number of switches with distance $i$ hops away from switch $u$ can be recursively bounded by $y_{i} \leq$ $(R-H-1) y_{i-1}=(R-H-1)^{i-1}(R-H)$, as each $i$-th switch has one port connecting to $(i-1)$-th switch. Since there are total $N / H$ switches, the number of switches with at least $m$ hops away from switch $u$ is $\frac{N}{H}-1-\sum_{i=1}^{m-1} y_{i}$ and is at least

$$
\frac{N}{H}-1-\sum_{i=1}^{m-1} y_{i} \geq \frac{N}{H}-1-(R-H) \frac{(R-H-1)^{m-1}-1}{R-H-2}
$$

Algorithm 1 generates a traffic matrix with high pair-wise shortest path length. In each iteration (Line 3-7), from unpicked switches, it arbitrarily picks a switch $u$ and then a switch $v$ which maximizes the shortest path length from $u$ (Line 4). Then, it updates entries $t_{u v}$ and $t_{v u}$ of the traffic matrix $T$ with $H$.

LEMMA 8.2. Given a uni-regular topology with total servers $N$ and $H$ servers per switch, Algorithm 1 constructs a traffic matrix with at least $W_{m}$ non-zero entries whose shortest path lengths are at least $m$, for $m \in\{1, \ldots, d\}$.

Proof. We will show that there are at least $W_{m}$ non-zero entries whose shortest path lengths are at least $m$ at the end of $k_{m}$-th iteration of Algorithm 1 for every $m$. Fix $m$ and $W_{m}$ from Lemma 8.1. Let $Q_{k}$ be the set of switches already picked after $k$-th iteration and $Q_{0}=\emptyset$. In the $k$-th iteration, switches $u$ and $v$ are picked from unpicked switches in $\mathcal{K} \backslash Q_{k-1}$ such that $v$ maximizes the shortest path length from $u$. Let $\mathcal{V}_{m}^{u}$ denote the set of switches with distance of at least $m$ hops from switch $u$. We observe that (a) if $\left|\mathcal{V}_{m}^{u} \backslash Q_{k-1}\right|$ is non-empty, $v$ will be picked from $\mathcal{V}_{m}^{u} \backslash Q_{k-1}$; (b) if $W_{m}-2(k-1)>0$, then $\mathcal{V}_{m}^{u} \backslash Q_{k-1}$ is non-empty because $\left|\mathcal{V}_{m}^{u} \backslash Q_{k-1}\right| \geq\left|\mathcal{V}_{m}^{u}\right|-\left|Q_{k-1}\right| \geq W_{m}-2(k-1)>0$. (We use Lemma 8.1 that $\left|\mathcal{V}_{m}^{u}\right| \geq W_{m}$ and the fact that $\left|Q_{k-1}\right|=2(k-1)$.)
Then, we choose $k_{m}=\left\lfloor\left(W_{m}+1\right) / 2\right\rfloor$, which always exists because $W_{m}$ is monotonically decreasing and at the highest $W_{1}=|\mathcal{K}|-1$, the chosen $k_{1}=\lfloor|\mathcal{K}| / 2\rfloor$ is feasible. Therefore, in the $k_{m}$-th iteration, we have $W_{m}-2\left(k_{m}-1\right)>0$ (satisfying (b)), so $\left|\mathcal{V}_{m}^{u} \backslash Q_{k-1}\right|$ is nonempty (satisfying (a)), and $v$ is picked from $\mathcal{V}_{u}^{m}$. Thus, at the end of the iteration, there are $2 k_{m}$ pairs and all of them have shortest path lengths at least $m$ since they are selected from $\bigcup_{u \in \mathcal{K}} \mathcal{V}_{m}^{u}$. Further, their number is at least $W_{m}$ because $2 k_{m}=2\left\lfloor\left(W_{m}+1\right) / 2\right\rfloor \geq$ $W_{m}$.

Lemma 8.3. Given a uni-regular topology with total servers $N$ and $H$ servers per switches, a traffic matrix $T$ constructed from Algorithm 1 has the following property:

$$
\max _{T^{\prime} \in \hat{\mathcal{T}}} \sum_{(u, v) \in \mathcal{K}^{2}} L_{u v} \mathbb{I}\left[t_{u v}^{\prime}>0\right] \geq \sum_{m=1}^{d} W_{m},
$$

where $W_{m}$ for $m \in\{1, \ldots, d\}$ is defined in Lemma 8.1 and $d$ is the minimum diameter from Moore bound [39].

Proof. Since the traffic matrix $T$ constructed from Algorithm 1 is a permutation traffic matrix, it follows that

$$
\max _{T^{\prime} \in \hat{\mathcal{T}}} \sum_{(u, v) \in \mathcal{K}^{2}} L_{u v} \mathbb{I}\left[t_{u v}^{\prime}>0\right] \geq \sum_{(u, v) \in \mathcal{K}^{2}} L_{u v} \mathbb{I}\left[t_{u v}>0\right] .
$$

It remains to show that $\sum_{(u, v) \in \mathcal{K}^{2}} L_{u v} \mathbb{I}\left[t_{u v}>0\right] \geq \sum_{m=1}^{d} W_{m}$. In the traffic matrix $T$, let $\mathcal{V}_{m}$ be the set of switch pairs whose shortest path lengths are at least $m$ hops. From the definition, we know that $\mathcal{V}_{d} \subseteq \mathcal{V}_{d-1} \subseteq \ldots \subseteq \mathcal{V}_{1}$, and $\mathcal{V}_{m} \backslash \mathcal{V}_{m+1}$ only contains switch pairs with exactly $m$ hops for $m \in\{1, \ldots, d-1\}$. It follows that

$$
\begin{aligned}
\sum_{(u, v) \in \mathcal{K}^{2}} L_{u v} \mathbb{I}\left[t_{u v}>0\right] \geq d\left|\mathcal{V}_{d}\right|+\sum_{m=1}^{d-1} m\left|\mathcal{V}_{m} \backslash \mathcal{V}_{m+1}\right| \\
\geq d\left|\mathcal{V}_{d}\right|+\sum_{m=1}^{d-1} m\left(\left|\mathcal{V}_{m}\right|-\left|\mathcal{V}_{m+1}\right|\right)=\sum_{m=1}^{d}\left|\mathcal{V}_{m}\right|
\end{aligned}
$$

Applying the fact that $\left|\mathcal{V}_{m}\right| \geq W_{m}$ for every $m \in\{1, \ldots, d\}$ from Lemma 8.1, we have

$$
\sum_{(u, v) \in \mathcal{K}^{2}} L_{u v} \mathbb{I}\left[t_{u v}>0\right] \geq \sum_{m=1}^{d} W_{m}
$$

\section{Proof of Theorem 4.1.}

Proof. To prove this theorem, we apply Lemma 8.1 and Lemma 8.3 to the RHS of Theorem 2.2. We have;

$$
\begin{aligned}
\theta^{*} & \leq \min _{T \in \hat{\mathcal{T}}} \frac{2 E}{H \sum_{(u, v) \in \mathcal{K}^{2}} L_{u v} \mathbb{I}\left[t_{u v}>0\right]} \\
& =\frac{2 E}{H \max _{T \in \hat{\mathcal{T}}} \sum_{(u, v) \in \mathcal{K}^{2}} L_{u v} \mathbb{I}\left[t_{u v}>0\right]} \leq \frac{2 E}{H D}
\end{aligned}
$$

where

$$
D=\sum_{m=1}^{d} W_{m}=d\left(\frac{N}{H}-1\right)-\frac{R-H}{R-H-2}\left(\frac{(R-H-1)^{d}-1}{R-H-2}-d\right)
$$


From Equation 12 and using the fact that in uni-regular topologies, $2 E=\frac{N}{H}(R-H)$, we have the upper bound in Equation 2.

\section{E Asymptotic behavior of throughput gap}

In §3.1, we pointed out that the throughput gap for Jellyfish might be expected to be non-zero in the range $100 \mathrm{~K}-180 \mathrm{~K}$ servers, but could not confirm this because our KSP-MCF implementation does not scale to these sizes. To be able to quantify the throughput gap for topologies larger than our computational limit for KSP-MCF, we compute a lower bound on throughput when routing can exploit all paths of length equal to or less than the length of the shortest path plus $M$ ( $M$ is a parameter to the lower bound calculation) in Theorem 8.4. Define the theoretical throughput gap to be the difference between the upper and lower bounds (for a given $M$ ). Intuitively, the theoretical throughput gap shows the maximum possible gap one can expect when using our bound in Theorem 2.2. Figure A.1 shows that the magnitude of the theoretical gap as a function of the topology size. (we use $M=1$; at this setting, each topology has at least 300 distinct paths between each source-destination pair across the entire range of topology sizes we have considered, which is sufficient for our path-based MCF computation $\S \mathrm{H}$ ).

Figure A. 1 shows that the maximum possible gap at these scales is going to be smaller than that of $3 \mathrm{~K}-15 \mathrm{~K}$. Moreover, the theoretical gap decreases as the size of the topology grows. We prove this observation in Corollary 2 showing that the theoretical throughput gap approaches zero asymptotically. In other words, for very large topologies, we expect our throughput bound to match the actual topology throughput.

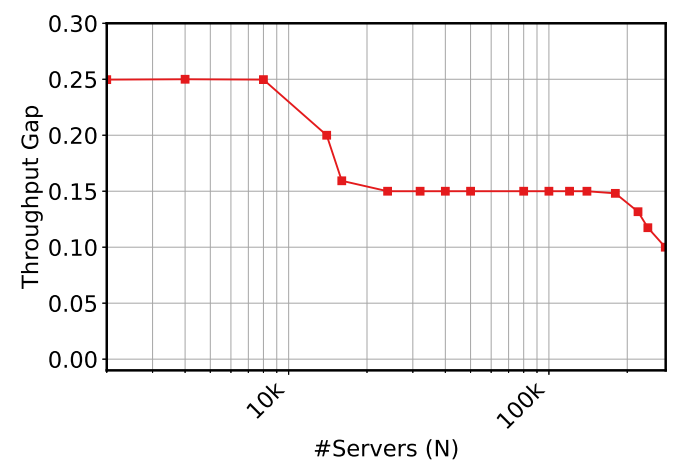

FiguRE A.1: Theoretical throughput gap.

We first start by stating the following assumption that always holds in all of our experiments.

Assumption 1. Given a traffic matrix $T$ and a corresponding solution of our path-based MCF, the ingress capacity of networkfacing ports is saturated by traffic at every switch:

$$
\begin{aligned}
& X_{u}(T)+\theta(T) \sum_{v \in \mathcal{K} \backslash\{u\}} t_{v u}=R_{u}-H_{u} \quad \text { for every } u \in \mathcal{K} \\
& X_{u}(T)=R_{u} \quad \text { for every } \quad u \in \mathcal{S} \backslash \mathcal{K},
\end{aligned}
$$

where $X_{u}(T)$ is the amount of transit traffic on switch $u$ as a result of routing the traffic matrix $T$. Note that $H_{u}=0$ for every switch with no servers, and it is omitted in the second equality.

Intuitively, the assumption holds in practice because datacenter topologies are designed such that all the link capacities can be fully utilized, as are the ingress capacities. We use this assumption to prove a bound on throughput gap. Let $M$ denote the additive path length such that every path length is bounded by

$$
\operatorname{len}(p) \leq L_{u v}+M \text { for every } p \in \mathcal{P}_{u v} \text {, and every }(u, v) \in \mathcal{K}^{2} .
$$

THeOREM 8.4. Under a permutation traffic matrix $T \in \hat{\mathcal{T}}$, when Assumption 1 holds with the additive path length $M_{T}$ (depending on $T$ ), the maximum achievable throughput of a topology (either uni-regular or bi-regular) is at least;

$$
\theta(T) \geq \frac{2 E}{N M_{T}+H \sum_{(u, v) \in \mathcal{K}^{2}} L_{u v} \mathbb{I}\left[t_{u v}>0\right]} .
$$

Proof. Let $\mathcal{S}$ denote the set of all switches. From Assumption 1 , we sum the transit traffic $X_{u}(T)$ over all switches and have the following equality

$$
\sum_{u \in \mathcal{S}} X_{u}(T)=\sum_{u \in \mathcal{S}}\left(R_{u}-H_{u}\right)-\theta(T) \sum_{u \in \mathcal{K}} \sum_{v \in \mathcal{K} \backslash\{u\}} t_{v u}
$$

Note that Assumption 1 changes the inequality in Equation 9 to equality due to all ingress capacity is fully utilized.

Alternatively, we can compute the total transit traffic $\left(\sum_{u \in \mathcal{S}} X_{u}(T)\right)$ based on Equation 5;

$$
\sum_{u \in \mathcal{S}} X_{u}(T)=\theta(T) \sum_{u \in \mathcal{K}} \sum_{v \in \mathcal{K} \backslash\{u\}} t_{u v} \sum_{p \in \mathcal{P}_{u v}} \beta_{p}(T)(\text { len }(p)-1) .
$$

Since length of all the paths in $\mathcal{P}_{u v}$ is at most $L_{u v}+M_{T}$ from the definition of the additive path length, we have;

$$
\sum_{u \in \mathcal{S}} X_{u}(T) \leq \theta(T) \sum_{u \in \mathcal{K}} \sum_{v \in \mathcal{K} \backslash\{u\}} t_{u v}\left(L_{u v}+M_{T}-1\right) .
$$

From Equation 14 and Equation 15, we have

$$
\theta(T) \geq \frac{\sum_{u \in \mathcal{S}}\left(R_{u}-H_{u}\right)}{\sum_{u \in \mathcal{K}} \sum_{v \in \mathcal{K} \backslash\{u\}} t_{u v}\left(L_{u v}+M_{T}\right)} .
$$

Finally, using the fact that a) $\sum_{u \in \mathcal{S}}\left(R_{u}-H_{u}\right)=2 E$, b) $T$ is a permutation traffic matrix, c) $L_{u u}=0$ for every switch $u$ and d) the sum of all the entries except the diagonals of the traffic matrix $T$ is at most $N$, we can derive the throughput lower bound in Equation 13.

The above theorem states the lower bound of throughput with respect to the additive path length $M_{T}$ depending on a given permutation traffic matrix $T$. Our path-based MCF computation shows that $M_{T}=1$ is sufficient to provide enough path diversity to make Assumption 1 valid for all Jellyfish, Xpander and FatClique. Using Theorem 8.4, we show that the gap between the upper bound and the lower bound can be arbitrarily small when the network size is sufficiently large and when a mild assumption holds.

Assumption 2. The additive path length for the maximal permutation traffic matrix $\hat{T}$ does not increase with a topology size such that $M_{\hat{T}}=O(1)$. 
Corollary 2. When Assumptions 1 and 2 hold, for any positive value $\epsilon>0$, any uni-regular topology with $N$ servers has $N_{\epsilon}^{*}$ such that for every $N \geq N_{\epsilon}^{*}$;

$$
\theta^{*}-\theta_{l b} \leq \epsilon
$$

where $\theta^{*}$ is the throughput upper bound from Theorem 2.2 and $\theta_{l b}=$ $\min _{T \in \hat{\mathcal{T}}} \theta_{l b}(T)$ is the mininum of throughput lower bound $\theta_{l b}(T)$ from Theorem 8.4.

Proof. From Assumption 1, it holds for every permutation matrix $T \in \hat{\mathcal{T}}$ that

$$
\begin{aligned}
& \theta^{*}-\theta_{l b}(T) \leq \theta^{*}- \min _{T \in \hat{\mathcal{T}}} \theta_{l b}(T) \leq \\
& \theta^{*}-\min _{T \in \hat{\mathcal{T}}} \frac{2 E}{N M_{T}+H \sum_{(u, v) \in \mathcal{K}^{2}} L_{u v} \mathbb{I}\left[t_{u v}>0\right]} .
\end{aligned}
$$

Let $\hat{T}=\left[\hat{t}_{u v}\right]$ be the maximal traffic matrix that minimizes the right side of Equation 1. We observe that it also minimizes the last term above, and we have

$$
\begin{aligned}
& \theta^{*}-\theta_{l b}(T) \leq \\
& \quad \frac{2 E N M_{\hat{T}}}{\left(N M_{\hat{T}}+H \sum_{(u, v) \in \mathcal{K}^{2}} L_{u v} \mathbb{I}\left[\hat{t}_{u v}>0\right]\right)\left(H \sum_{(u, v) \in \mathcal{K}^{2}} L_{u v} \mathbb{I}\left[\hat{t}_{u v}>0\right]\right)} .
\end{aligned}
$$

Using Lemma 8.3 and Lemma 8.1, we have;

$$
\begin{aligned}
& \sum_{(u, v) \in \mathcal{K}^{2}} L_{u v} \mathbb{I}\left[\hat{t}_{u v}>0\right] \geq \\
& d\left(\frac{N}{H}-1\right)-\frac{R-H}{R-H-2}\left(\frac{(R-H-1)^{d}-1}{R-H-2}-d\right)=D .
\end{aligned}
$$

Equation 16 and Equation 17 lead to

$$
\theta^{*}-\theta_{l b}(T) \leq \frac{2 E N M_{\hat{T}}}{\left(N M_{\hat{T}}+H D\right)(H D)}
$$

Since the above inequality holds for every $T \in \hat{\mathcal{T}}$, it holds at the worst-case gap

$$
\theta_{u b}-\min _{T \in \hat{\mathcal{T}}} \theta_{l b}(T) \leq \frac{2 E N M_{\hat{T}}}{\left(N M_{\hat{T}}+H D\right)(H D)} .
$$

Similar to Corollary 1 , we can prove that above inequality goes to 0 as $N$ increases because every $M_{T}$ is bounded by a constant independent of $N$ under Assumption 2.

\section{F Throughput of bi-regular Clos topologies under TUB}

TUB is tight for bi-regular Clos topologies as well, giving throughput equal to 1 for different topology sizes (Table A.1).

\begin{tabular}{|c|c|c|c|}
\hline $\mathrm{N}$ & \#Layers & \#SWs & TUB \\
\hline 8192 & 3 & 1280 & 1.00 \\
32768 & 4 & 7168 & 1.00 \\
131072 & 4 & 28672 & 1.00 \\
\hline
\end{tabular}

TABLE A.1: Clos: tub is always 1.

\section{G Proof of Corollary 1}

Proof. This follows directly from Equation 2 in Theorem 4.1. We can show that, in $D$, the term containing $N d$ dominates the other terms for large enough $N$. This is a direct consequence of defining $d$ as the minimum diameter that required to accommodate $\mathrm{N} / \mathrm{H}$ switches (Moore bound [39]). As a result, in the RHS of the Equation 2, the numerator grows as $N$ and the denominator grows as $N d$. Therefore, $\theta^{*}$ approaches zero with increasing $N$, so there must always exist a $N^{*}$ at which $\theta^{*}$ falls below 1 .

\section{H Path-based Multi-commodity Flow LP formulation}

In this section, we briefly introduce the path-based MCF formulation (common in WAN traffic engineering [33]) used throughout the paper. Given a traffic matrix $T=\left[t_{u v}\right]$ and set of paths between every pair of switches with servers $\left(\mathcal{P}_{u v}\right)$, the throughput of the traffic matrix is the solution to the following LP formula in which $f_{p}$ denotes the amount of flow on path $p$;

$$
\begin{array}{ll}
\operatorname{maximize} & \theta \\
\text { subject to } & \sum_{p \in \mathcal{P}_{u v}} f_{p} \geq \theta t_{u v} \quad \forall(u, v) \in \mathcal{K}^{2} \\
& \sum_{(u, v) \in \mathcal{K}^{2}} \sum_{p \in \mathcal{P}_{u v}} f_{p} \mathbb{I}[e \in p] \leq 1 \quad \forall e \in \mathcal{E} \\
& f_{p} \geq 0 \quad \forall(u, v) \in \mathcal{K}^{2}, \forall p \in \mathcal{P}_{u v},
\end{array}
$$

where $\mathcal{E}$ is the set of directional links with unit capacity.

\section{Metric Adjustments for FatClique}

In a FatClique, the number of servers attached to each switch can differ by at most 1 . To generalize the maximal permutation traffic matrix generation to accommodate this case, we changed weight assignment of edges in the complete bipartite graph from $w_{u \rightarrow v}=$ $L_{u v}$ to $w_{u \rightarrow v}=L_{u v} \min \left(H_{u}, H_{v}\right)$. The latter weight assignment takes into account the maximum amount of flow between each $u, v$ pair along with their distance. More precisely, if in a permutation traffic matrix $t_{u v}$ is non zero, it should be the minimum of $H_{u}$ and $H_{v}$ since it should conform to the hose-model traffic constraints $\S 2$. So, Equation 1 can be re-written as;

$$
\theta^{*} \leq \min _{T \in \hat{\mathcal{T}}} \frac{2 E}{\sum_{(u, v) \in \mathcal{K}^{2}} L_{u v} \min \left(H_{u}, H_{v}\right) \mathbb{I}\left[t_{u v}>0\right]}
$$

Equation 18 is exactly same as Equation 1 when all the switches have exactly the same $H$. To find the maximal permutation traffic matrix, we need to find the traffic matrix that minimizes the LHS of Equation 18. This is equivalent to solving the maximum weight matching in a bipartite graph (§2), with the revised weight assignment.

This approach does not yield the global minimum of the throughput bound since Theorem 2.1 does not hold when $\mathrm{H}$ differs accross the switches. A linear programming (LP) formulation can compute the global minimum [31]. However, we use our matching method to infer the maximal permutation traffic matrix for FatClique, for three reasons. First, in FatClique, the number of servers connected to each switch can differ only by 1 , so the difference between global minimum and throughput bound computed using this approach is negligible. Second, algorithms for solving maximal weight matching are more efficient than solving an LP. Third, the permutation traffic 


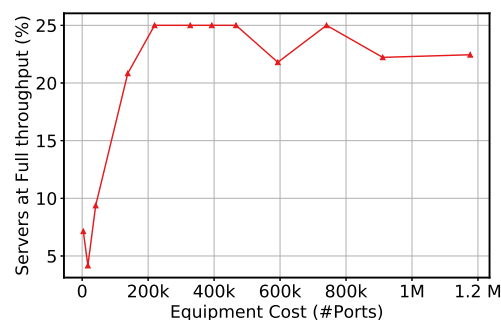

Figure A.2: Topology Cost (Jellyfish vs Fat-tree). The relative difference of the maximum servers supported at full throughput (per TUB) between Jellyfish and Fat-tree built with the same equipment using $\{14,24,32,48,56$, $64,68,72,78,84,90,98\}$-port switches averaged over 5 runs.

matrix generated using our approach is harder to route compared to an LP generated traffic matrix.

\section{J Throughput Gap for different values of $K$}

Figure A.5 illustrates the absolute difference between path-based multi-commodity flow over $K$-shortest paths and our throughput bound for different values of $K$ (i.e., throughput gap). The results for $K=60,100,200$ are very similar to each other; a gap of nonzero for small size topologies, followed by a close-to-zero gap for larger instances. The only exception is some instances of FatClique exhibit large throughput gaps in the $5 \mathrm{~K}-15 \mathrm{~K}$ compared to Jellyfish and Xpander because FatClique cannot fully utilize available capacity with $K=60,100$ for KSP-MCF. However, after increasing $K$ to 200 (Figure 5(1)), the throughput gap behavior for FatClique is comparable to Jellyfish and Xpander.

For $K=20$, the gap remains significant even at large topologies since 20-shortest paths does not provide enough diversity to completely exploit the network capacity, and some of the capacity remains unused.

\section{K Scaling of Throughput-based Cost Comparison}

Other than bisection bandwidth, Jellyfish [44] and Xpander [47] used full throughput of random permutations and all-to-all traffic matrices under MCF to assess the cost advantage of their topologies. However, throughput under random permutations and allto-all traffic matrices can be significantly larger than (worst-case) throughput [27]. Moreover, as discussed in §3.1, MCF and KSPMCF can not scale to the size of current datacenters. In this section, we show how conclusions can change when using our bound to perform cost comparisons at larger scale.

Jellyfish. Singla et al. [44] have shown that at the scale of $<900$ servers Jellyfish can support $27 \%$ more servers than a Fat-tree [1] built with same equipment, and conjecture that this cost advantage increases by using a higher radix switch. Figure A.2 shows the relative difference of the maximum servers between Jellyfish and Fat-tree for different switch radices. Using TUB, at the scale of 686 servers $(R=14$, which is the largest scale considered in [44]), Jellyfish can support only $8 \%$ more servers than a (same equipment) Fat-tree (the leftmost point in Figure A.2), dropping the cost advantage of Jellyfish by $3 \mathrm{x}$. Moreover, using a higher radix switch

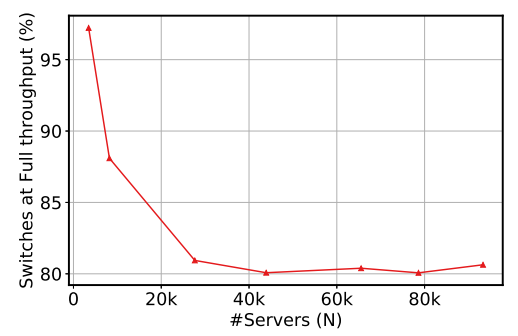

Figure A.3: Topology Cost (Xpander vs Fat-tree). Number of Switches required to support $N$ servers. Percentages are Xpander/Fat-tree.

does not result in higher cost advantage of Jellyfish over Fat-tree. In fact, using a higher radix switch might result in drop in the cost advantage. For example, using 98-port switches instead of 64-port causes the cost advantage to drop slightly from $25 \%$ to $22 \%$.

Xpander. Valadarsky et al. [47] have shown that at the scale of $<4 \mathrm{~K}$ servers, Xpander can support the same number of servers as Fat-Tree [1] at full throughput using $80 \%-85 \%$ of the switches. As Figure A.3 shows at the maximum considered scale in [47] (3.5K servers, the left most point), Xpander should use more than 95\% switches compared to the same size Fat-tree. However, as the scale grows, the cost advantage of Xpander over Fat-tree increases, matching the numbers reported in [47].

\section{Throughput of uni-regular topologies under expansion}

Jellyfish [44] and Xpander [47] have shown that using a very simple expansion algorithm (random rewiring), their design can be expanded to any size with minor throughput loss while preserving the number of servers per switch $H$. Jellyfish uses bisection bandwidth as their throughput metric while Xpander assesses the throughput by solving MCF on all-to-all traffic matrix.

Jellyfish. In §5.1, we show that Jellyfish requires advanced planning in order to preserve full throughput, otherwise, even very small expansion can turn Jellyfish into a topology with less than full throughput. To better understand the amount of throughput degradation, Figure A.4 shows the throughput (computing using TUB), normalized by the topologies initial throughput (before expansion). At each step, we expand the topology by $20 \%$ of the initial size until its size reaches the $2.6 \mathrm{x}$ of the initial topology. For $10 \mathrm{~K}$ servers, Figure A. 4 shows that throughput drops by more than $20 \%$ when expanding the topology by only $0.6 x$. On the other hand, when the initial topology size is $32 \mathrm{~K}$, throughput drop is negligible $(<1 \%)$. We emphasize that these results are consistent with $\S 4.2$; Jellyfish with $\mathrm{H}=6$ and initial size $8 \mathrm{~K}$ has full throughput even after expanding by 2.6x. However, it faces the throughput drop as well.

This suggests that operators should be cautious when expanding uni-regular topologies depending on the topology's initial and target size as they might face significant throughput drops. TUB, therefore, helps topology designers to identify and understand these scenarios before deploying and expanding their desired topology.

Xpander. Using tuB to assess the Xpander's performance under expansion results in similar conclusions as expanding Jellyfish does. Similar to Jellyfish, operators who adopt Xpander should have the 


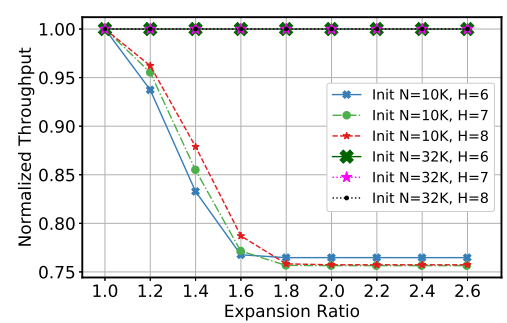

FIGURE A.4: Throughput of uni-regular topologies under expansion.

target size in mind and choose $H$ accordingly. Otherwise, they either end up having a topology with less than full throughput or have to rewire the servers, bearing a significant cost. The throughput degradation is also very similar to Jellyfish (Figure A.4); at some scales (e.g., 10K), expanding the Xpander even by a very small ratio degrades the throughput by as much as $25 \%$. 


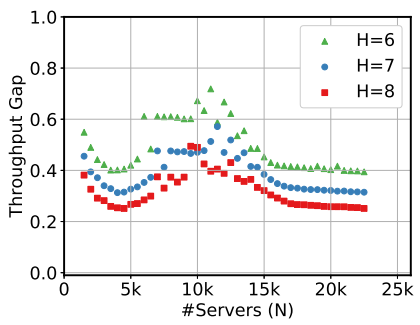

(a) Jellyfish, $\mathrm{K}=20$

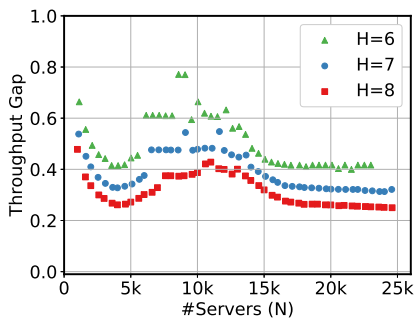

(e) Xpander, $\mathrm{K}=20$

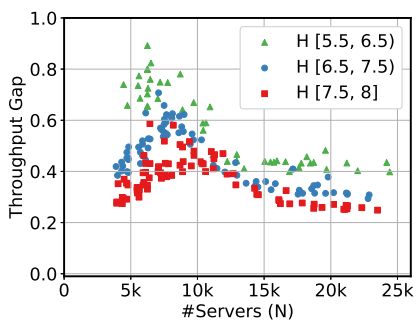

(i) FatClique, $\mathrm{K}=20$

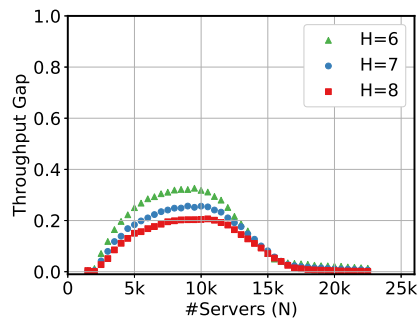

(b) Jellyfish, $\mathrm{K}=60$

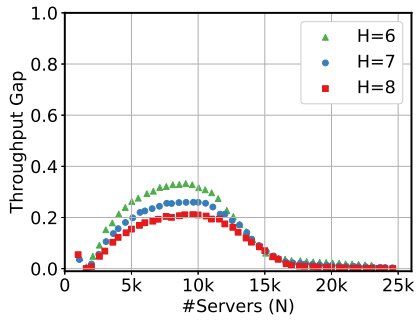

(f) Xpander, $\mathrm{K}=60$

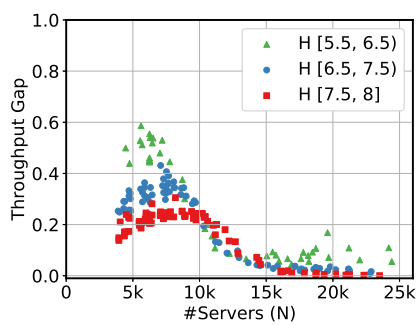

(j) FatClique, $K=60$

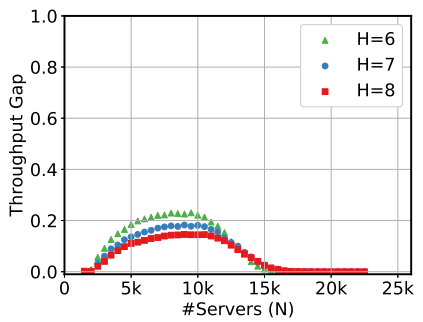

(c) Jellyfish, $\mathrm{K}=100$

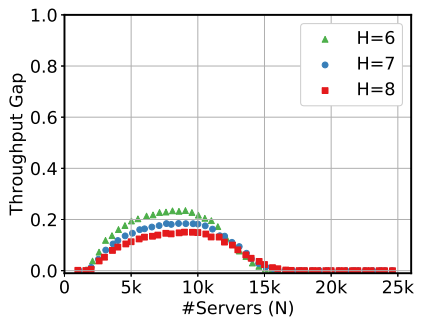

(g) Xpander, $\mathrm{K}=100$

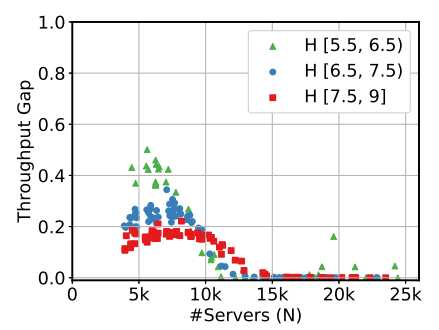

(k) FatClique, $\mathrm{K}=100$

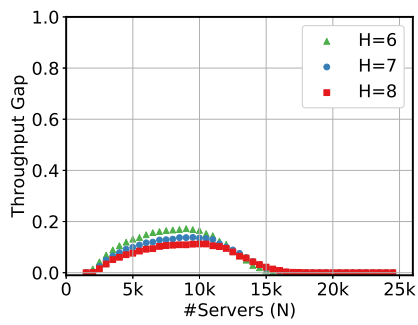

(d) Jellyfish, $\mathrm{K}=200$

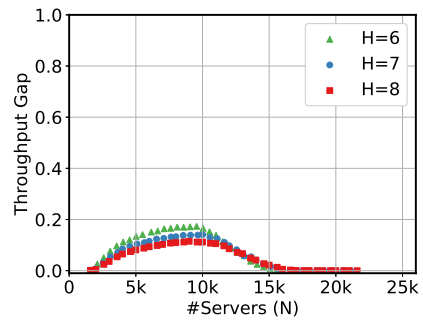

(h) Xpander, $\mathrm{K}=200$

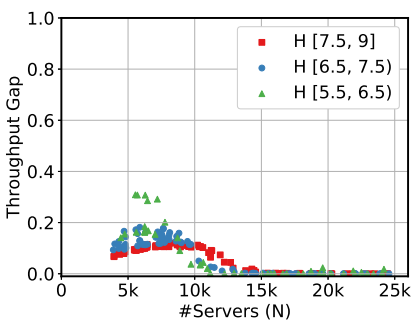

(l) FatClique, $K=200$

Figure A.5: Throughput bound vs K-shortest paths Multi-commodity flow for different values of $\mathrm{K}(20,60,100,200)$. 